\title{
LOCALITY AT THE BOUNDARY IMPLIES GAP IN THE BULK FOR 2D PEPS
}

\author{
Michael J. Kastoryano ${ }^{1,2}$, Angelo Lucia ${ }^{1,3,4}$, David Perez-Garcia ${ }^{5,6}$ \\ ${ }^{1}$ NBIA, Niels Bohr Institute, University of Copenhagen, Denmark \\ ${ }^{2}$ Institute for Theoretical Physics, University of Cologne, Germany \\ ${ }^{3}$ QMATH, Department of Mathematical Sciences, University of Copenhagen, Denmark \\ ${ }^{4}$ Walter Burke Institute for Theoretical Physics and Institute for Quantum Information \& Matter, \\ California Institute of Technology, Pasadena, CA 91125, US \\ ${ }^{5}$ Dpto. Análisis Matemático, Universidad Complutense de Madrid, 28040 Madrid, Spain \\ ${ }^{6} 5$ Instituto de Ciencias Matemáticas, 28049 Madrid, Spain
}

(Dated: December 18, 2018)

Proving that the parent Hamiltonian of a Projected Entangled Pair State (PEPS) is gapped remains an important open problem. We take a step forward in solving this problem by showing two results: first, we identify an approximate factorization condition on the boundary state of rectangular subregions that is sufficient to prove that the parent Hamiltonian of the bulk 2D PEPS has a constant gap in the thermodynamic limit; second, we then show that Gibbs state of a local, finite-range Hamiltonian satisfy such condition. The proof applies to the case of injective and MPO-injective PEPS, employs the martingale method of nearly commuting projectors, and exploits a result of Araki [1] on the robustness of one dimensional Gibbs states. Our result provides one of the first rigorous connections between boundary theories and dynamical properties in an interacting many body system.

\section{Contents}

\section{Introduction}

II. Preliminaries

A. PEPS basics

B. Boundary states of PEPS

C. The martingale condition and the spectral gap 8

III. The gap theorem for injective PEPS

A. Approximate factorization of the boundary states

B. Matrix Product States

IV. The gap theorem for non-injective PEPS

A. MPO-injectivity

B. Approximate factorization for MPO-injective PEPS

V. Approximate factorization of 1D thermal states

A. Local and quasi-local Hamiltonians

B. Analysis of 1D Gibbs states 20

C. Expansionals

D. The finite range Hamiltonian case

E. Extension to quasi-local interactions

F. Boundary Hamiltonians of PEPS

VI. Conclusion

Acknowledgments

\begin{tabular}{ll} 
References & 28 \\
\hline
\end{tabular} 


\section{INTRODUCTION}

Quantum Information Theory (QIT) and the Theory of Quantum Many Body (QMB) systems are inextricably connected. On the one hand, QMB systems and their properties, such as entanglement or topological order, play a crucial role in the design of quantum computers and quantum simulators. On the other hand, the use of QIT tools and ideas have shed new light on the structure, properties and mechanisms present in QMB systems.

An important part of these developments have come through the so called Tensor Networks States (TNS), variational families of states which mimic the entanglement structure present in ground states and thermal states of QMB systems. Indeed, as proven in a series of papers, Projected Entangled Pair States (PEPS), a particularly relevant family of TNS, approximate well ground and thermal states of local Hamiltonians [2-6]. Based on this, they have been used to design better algorithms to simulate QMB systems (see e.g. [7] for a recent review on this). A remarkable example in this direction is [8, 9], where a new algorithm to approximate ground states of gapped spin chains is presented and shown competitive compared to DMRG, while providing a guarantee of fast convergence.

Being arbitrarily close to any ground or thermal state of a QMB system, PEPS have been used also as a new analytical tool, based on QIT techniques, to give a rigorous mathematical treatment of some of the challenges posed by QMB systems. In this direction one can highlight for instance the classification given in [10-13] of (symmetry-protected) phases in 1D systems, or the microscopic explanation of topologically ordered systems of [14-18]. Since a PEPS is always the ground state of an associated short range Hamiltonian (called the parent Hamiltonian), this led for instance to the first local Hamiltonian having the Resonating Valence Bond (RVB) State as unique ground state in the kagome lattice (up to topological degeneracy) [19], a question that can be traced back to the seminal work of P. W. Anderson in the 70s [20]. Recently, TNS started to play also a key role in providing a rigorous framework to address some of the main open problems in high energy physics, such as those coming from Maldacena's AdS/CFT holographic correspondence [21-24].

A key insight in all these analytical developments is the existence of suitable connections between the bulk of the system and its boundary in every TNS. Such bulk-boundary correspondences have also played an important role in the study in QMB physics recently thanks to the seminal paper of $\mathrm{Li}$ and Haldane [25], where they observed that the spectrum of the reduced density matrix of a fractional quantum Hall ground state closely resembled a conformal field theory at the boundary [25]. This observation led the authors to conjecture that the entanglement spectrum contains information about the counting of edge states.

Following the path initiated by Li and Haldane, the bulk-boundary correspondence in PEPS was made explicit in [26] via an isometry and the definition of the so called boundary Hamiltonian. This is an auxiliary 1D interaction whose thermal state (called boundary state) has two key features: (1) its spectrum can be associated to the spectrum of the reduced density matrix of the given PEPS in the bulk and (2) its (boundary) correlations can be associated to the correlations present in the bulk.

Since thermal states of local, finite-range Hamiltonians have exponentially decaying correlations (a result due to Araki in [1]), the following conjecture was stated in [26]:

Conjecture 1. The bulk parent Hamiltonian of the PEPS is gapped if and only if the boundary Hamiltonian is short range.

This conjecture was verified numerically in [26] for Ising PEPS [27], one of the few models for which the bulk spectral gap is analytically proved. More generally, Conjecture 1 has led to new ways of numerically analyzing spectral gaps and quantum phase transitions in PEPSbased algorithms by looking at the boundary (see e.g. [28-32]).

Conjecture 1 also opens a new line of thought in the problem of characterizing analytically when a given 2D (or higher dimensional) PEPS has a gapped parent Hamiltonians. This 
problem has seen virtually no progress since the original AKLT paper (see however [33, 34]), even though it was stated as an important open problem in Ref. [35]. Its difficulty can be easily guessed from the recent result that proving spectral gaps in 2D short range Hamiltonians is undecidable [36]. Despite this, the problem is undoubtedly important. Apart from the potential solution of the long-standing spectral gap problem for the 2D AKLT Hamiltonian, a sufficiently sharp condition for the existence of a spectral gap in PEPS Hamiltonians seems crucial to attack two other central open problems in QMB systems.

The first one is a mathematically complete classification of gapped quantum phases in 2D, at least for the cases which can be characterized well within the PEPS framework (groundstates of gapped non-chiral Hamiltonians are believed to have an efficient description in terms of PEPS, while the situation for gapped chiral models is less clear [37-40]). Let us recall that two Hamiltonians are said to be in the same phase if they can be deformed into each other without crossing a phase transition point (i.e. closing the gap). Recent work on Renormalization Fixed Points (RFP) [17] allows to conclude then (up to subtle but important technical details) that essentially only Levin-Wen string-net models [41] appear as 2D RFP. The main remaining step is then to connect with a gapped path of (parent) Hamiltonians each PEPS with one of the RFP models. Clearly this can only be done if one finds a suitable criterion to guarantee the presence of such gap.

The second potential application of a sharp gap criterion is a mathematical proof of the existence a gapped $S U(2)$-invariant topological spin liquid phase. Topological spin liquids constitute a long-sought new quantum phase that can be traced back to the revolutionary work of Anderson [20], and for which experimental evidence has been observed, even in naturally occurring materials such as herbertsmithite, although in a gapless form [42]. They are characterized by the absence of order even at zero temperature, and in the case of gapped spin liquids they are expected to show topological order and quasi-particle excitations [43]. A long standing conjecture regards the existence of a gapped spin liquid which does not break a local $S U(2)$ symmetry, for which the most promising candidate is the RVB state. The only remaining step to prove is to show that the parent Hamiltonian for the RVB constructed in [19] is in the same phase as the Toric Code. An interpolating path of parent Hamiltonians connecting the Toric Code and the RVB is constructed in [19] and numerical evidence is provided that no phase transition occur along the path. But the lack of a suitable gap characterization makes it hard to prove analytically the existence of a gap throughout the path.

In this paper, we take a first important step in solving the PEPS gap problem following the approach of Conjecture 1. We identify one condition on the boundary state of a $2 \mathrm{D}$ injective PEPS, which we call approximate factorization, and show that this condition is sufficient to prove a spectral gap in the bulk.

Main Result 1. If for any rectangular subregion of the lattice, the boundary state on the virtual indices of an injective $2 D$ PEPS is approximately factorizable then the gap of the bulk parent Hamiltonian is bounded below by a constant, in the thermodynamic limit.

The result can be extended to topologically ordered systems described by MPO-injective PEPS in a natural way. In this case, the boundary Hamiltonian is given by a sum of quasi-local terms plus one global projector that commutes with all other terms, and specifies the topological sector, and the approximate factorization condition is required on the non-topological part of the boundary state.

The proof strategy of Main Result 1 is based on the so-called martingale method: a general strategy that relates the gap of a frustration free Hamiltonian to the approximate commutativity of overlapping projectors. The martingale method is inspired from the classical proofs for showing rapid mixing of Glauber dynamics of Ising type models at finite temperature [44]. Nachtergaele first adapted this strategy for proving the gap of one dimensional VBS models [33]. Here we use a slightly different version of the proof due to Bertini et al. [45] which was generalized for frustration free Hamiltonians in [46] and for commuting quantum Gibbs 
samplers in [47]. Subsequently, the martingale condition was shown to be implied by the gap [46, 47]. The basic idea underlying the proof is to show that when we double the system size, the gap remains almost unchanged. Then one can grow the size indefinitely while keeping the gap constant. In this paper, we will prove the version of the martingale condition (Eqn. (10p) due to Bertini et al. (see Fig. 33 and refer back to Ref. [46] for the details of how this condition implies a gap of the parent Hamiltonian in the thermodynamic limit.

In the setting of Glauber dynamics for classical spin systems, the martingale condition on the ground state projectors can be rather easily shown to follow from decay of correlations in the bulk Gibbs state because of the (Dobrushin-Lanford-Ruelle) DLR theory of boundary conditions for classical Gibbs states [48, 49]. The DLR theory roughly states that the set of reduced states of all global Gibbs states restricted to a region is equal to the set of all local Gibbs states on that region with arbitrary boundary conditions. Since this set is convex, one can further restrict attention to states with pure boundary conditions, which in the classical setting are product states. Unfortunately, the DLR theory breaks down in the quantum setting both for proving gaps of quantum Gibbs samplers and for proving gaps of frustration free Hamiltonians [50]. Therefore the connection between bulk correlations and the martingale condition is lost. In short, the dynamic vs. static equivalence that the martingale condition provides in the classical setting is lost in the quantum setting. Main Result 1 shows that for PEPS this equivalence can be partially recovered: for injective PEPS, proving the martingale condition reduces to showing that the boundary states on the rectangular regions of Fig. 3 can be decomposed in a special way that we call approximate factorization. Approximate factorization of a state $\rho_{A B C}$ on a line $A B C$ broken up such that $B$ separates $A$ from $C$, claims that the state can be written as $\rho_{A B C} \approx \Omega_{A B} \Sigma_{B C}$ for operators $\Omega_{A B}$ and $\Sigma_{B C}$ with support on $A B$ and $B C$ respectively (Eqns. (13/14|15p). The actual condition of approximate factorization in Def. 9 is more complicated, and involves several boundary states on rectangular regions as in Fig. 4

We then show that locality of the interactions in the boundary Hamiltonian is sufficient to prove the approximate factorization condition.

Main Result 2. Gibbs (thermal) states of a one dimensional local finite-range Hamiltonian are approximately factorizable.

The proof of Main Result 2 is based on a careful use and generalization of the techniques developed by Araki in [1]. The limitation on faster than exponentially decaying interactions arises from the generalization of these techniques, which we are only able to prove for this case. By proving a generalization of Main Result 2 to the case of exponentially decaying interactions, which are the ones suggested by the numerical evidence, one would obtain a proof of one half of Conjecture 1. namely that a short range boundary Hamiltonian implies a bulk gap. Whether Main Result 2 admits such generalization is one of the open questions left in this paper. We will show how one can extend Main Result 2 to interactions decaying faster than any exponential, if one assumes that Araki's theorem holds in this case. In the following we will call such interactions quasi-local. Improving this result to a plain exponential, if true, seems a hard task since it is related with the believed but unproven fact that thermal states of 1D short range Hamiltonians with exponentially decaying interactions have exponential decay of correlations.

The paper is organized as follows. In Sec. II we introduce the notation and overview the basics of PEPS including the construction of the parent Hamiltonian. We also define boundary states of PEPS for rectangular regions of the lattice, and we introduce the equivalence between the martingale condition and the spectral gap of the parent Hamiltonian of the PEPS [46]. In Sec. III] we show that, for injective PEPS, the martingale condition can be reduced to the approximate factorization property of the boundary states on rectangular regions. Moreover, we consider the case of a PEPS defined on a 1D chain, which are known as Matrix 
Product States (MPS). We show that the boundary states of injective MPS are always approximately factorizing, providing an independent proof that the parent Hamiltonian of injective MPS is gapped [51]. In Sec. IV], we show how our main theorem relating approximate factorization to the martingale condition can be extended to MPO-injective PEPS in a natural way. Sec. V is devoted to the Main Result 2, namely that for Gibbs states of a one dimensional finite-range local Hamiltonian (not to be confused with the parent Hamiltonian of the PEPS) the approximate factorization property holds. This section requires a number of tools and results on the analysis of Gibbs states of local Hamiltonians on a line [1]. In order to compare states on different overlapping regions, we need to introduce the assumptions of locality and homogeneity on the Hamiltonian. Finally, we show how an extension of Araki's theorem to the case of quasi-local interaction, which we conjecture holds but we do not provide a proof of, can be straightforwardly used to generalize our results to this more general class of interactions. In the conclusion, we discuss further problems and implications of our theorem, as well as the relationship between exponential decay of correlations in the bulk and the fact that the boundary states are quasi-local and quasi-homogeneous Gibbs states.

\section{PRELIMINARIES}

\section{A. PEPS basics}

We will consider $\Lambda$ to be a finite subset of an infinite graph $(G, E)$, which can be isometrically embedded in $\mathbb{R}^{2}$ (the standard examples being the square lattice $\mathbb{Z}^{2}$ or the honeycomb lattice). At each $u \in G$ we will associate a finite-dimensional Hilbert space $\mathcal{H}_{d}$ of some fixed dimension $d$. We will denote by $\mathcal{H}_{\Lambda}=\bigotimes_{u \in \Lambda} \mathcal{H}_{d}$ the Hilbert space associated to $\Lambda$, and $\mathcal{B}\left(\mathcal{H}_{\Lambda}\right)$ is the set of bounded operators on $\mathcal{H}_{\Lambda}$. If $\left\{\left|j_{i}\right\rangle\right\}_{i=1}^{d}$ is an orthonormal basis for the Hilbert space at site $j$, any pure state in $\mathcal{H}_{\Lambda}$ can be written as

$$
|\psi\rangle=\sum_{j_{1}, \cdots, j_{N}=1}^{d} R_{j_{1}, \cdots, j_{N}}\left|j_{1}\right\rangle \otimes \cdots \otimes\left|j_{N}\right\rangle,
$$

where $N=|\Lambda|$ and $R_{j_{1}, \cdots, j_{N}}$ is the vector of amplitudes of $|\psi\rangle$, which we can think of as a tensor in $\left(\mathcal{H}_{d}^{*}\right)^{\otimes N}$.

Projected Entangled Pair States (PEPS) are a class of pure states for which it is possible to find a special description of the tensor $R$. They are constructed by associating to each edge $e \in E$ a maximally entangled state $\left|\omega_{e}\right\rangle=D^{-1 / 2} \sum_{j=1}^{D}|j, j\rangle$, where $D$ is called the bond dimension, and to each vertex $v \in G$ a linear map $T_{v}: \mathcal{H}_{D}^{\otimes r} \rightarrow \mathcal{H}_{d}$, where $r$ is the degree of the vertex $v$ (the number of edges incident to $v$ ). The Hilbert space associated with the edges is called the virtual space, whereas the one associated to vertices is called the physical space. $T_{v}$ can be considered as a map from the virtual space associated to the edges $e$ connected to $v$, onto the physical space at $v$ :

$$
T_{v}=\sum_{k_{v}=1}^{d} \sum_{j_{1}, \cdots, j_{r}=1}^{D} T_{j_{1}, \cdots, j_{r}}^{k_{v}}\left|k_{v}\right\rangle\left\langle j_{1}, \cdots, j_{r}\right| .
$$

Then $\left(T_{j_{1}, \cdots, j_{r}}^{k_{v}}\right)$ is a tensor with one physical index and $r$ virtual indices. On a square lattice, as in Fig. 11 $r=4$. If $\Lambda$ has no outgoing edges, then we can define a state in $\mathcal{H}_{\Lambda}$ via a tensor contraction:

$$
\left|\operatorname{PEPS}_{\Lambda}\right\rangle=\bigotimes_{v \in \Lambda} T_{v} \bigotimes_{e \in E_{\Lambda}}\left|\omega_{e}\right\rangle
$$


a)

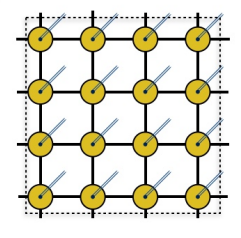

$A$ b)

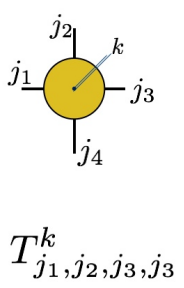

c)

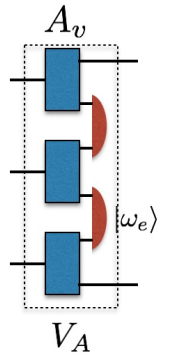

FIG. 1: We consider a PEPS state on a square lattice. a) Section of the PEPS on region $A \subseteq \Lambda$, b) Graphical representation of the tensors $T_{j_{1}, j_{2}, j_{3}, j_{4}}^{k}$, c) representation of the operator $V_{A}$ on a one dimensional lattice. The operator is to be read as mapping virtual indices (from the right) to physical indices (to the left).

If instead there are edges connecting $\Lambda$ with its complement in $(G, V)$, then we obtain a state in $\mathcal{H}_{\Lambda}$ for each choice of "boundary condition", in the following sense: denote with $E_{\bar{\Lambda}}$ the edges which are incident to $\Lambda$, with $E_{\Lambda}$ the edges with are contained in $\Lambda$, and with $\partial \Lambda=E_{\bar{\Lambda}} \backslash E_{\Lambda}$ the edges that connect $\Lambda$ with its complement. Let $\mathcal{H}_{\partial \Lambda}=\bigotimes_{e \in \partial \Lambda} \mathcal{H}_{D}$ (note that while at each edge we associated $\left|\omega_{e}\right\rangle \in \mathcal{H}_{D} \otimes \mathcal{H}_{D}$, we are only including one copy of $\mathcal{H}_{D}$ in $\left.\mathcal{H}_{\partial \Lambda}\right)$. Then for each vector $|X\rangle \in \mathcal{H}_{\partial \Lambda}$ we can define a state

$$
\left|\operatorname{PEPS}_{\Lambda, X}\right\rangle=\left\langle X\left|\bigotimes_{v \in \Lambda} T_{v} \bigotimes_{e \in E_{\bar{\Lambda}}}\right| \omega_{e}\right\rangle
$$

This defines a linear map from $\mathcal{H}_{\partial \Lambda}$ to $\mathcal{H}_{\Lambda}$, which we will denote with $V_{\Lambda}$. It is a mapping from the virtual indices at the boundary of $\Lambda$ to the physical indices in the bulk of $\Lambda$ (see Fig. 1 for an illustration):

$$
\begin{aligned}
V_{\Lambda}: \mathcal{H}_{\partial \Lambda} & \rightarrow \mathcal{H}_{\Lambda} \\
|X\rangle & \mapsto\left|\operatorname{PEPS}_{\Lambda, X}\right\rangle .
\end{aligned}
$$

A PEPS is said to be injective on $\Lambda$ [52] if $V_{\Lambda}$ is an injective map. As shown in Ref. [52], if a PEPS is injective on disjoint regions $A$ and $B$, it is also injective on $A \cup B$, so we will simply assume, up to coarse graining of the lattice, that $V_{\Lambda}$ is injective for every finite $\Lambda$.

Again following Ref. [52], for any injective PEPS, we can define a local Hamiltonian, called the parent Hamiltonian, for which the PEPS is the unique groundstate. This is done by considering, for each edge $e=(a, b)$, the orthogonal projector $h_{e}$ on the orthogonal complement of $\operatorname{Im} V_{\{a, b\}}$. Then $H_{\Lambda}=\sum_{(a, b) \in E_{\Lambda}} h_{e}$ is a local Hamiltonian, and clearly $H_{\Lambda}\left|\mathrm{PEPS}_{\Lambda, X}\right\rangle=0 . H_{\Lambda}$ is frustration-free: i.e. $h_{e}\left|\mathrm{PEPS}_{\Lambda, X}\right\rangle=0$ for all $e \in E_{\Lambda}$.

It will be very important for us to talk about sub-regions of the lattice $A \subseteq \Lambda$, and to consider the associated local ground subspace $\left.\mathcal{G}_{A}=\left\{|\varphi\rangle \in \mathcal{H}_{\Lambda}\left|H_{A}\right| \phi\right\rangle=0\right\}=\operatorname{Im} V_{A}$, for $H_{A}=\sum_{e \in E_{A}} h_{e}$. We will denote with $P_{A}$ the orthogonal projector on $\mathcal{G}_{A}$. Because of frustration freeness, for any $A \subseteq B \subseteq \Lambda$, we have $\mathcal{G}_{\Lambda} \subseteq \mathcal{G}_{B} \subseteq \mathcal{G}_{A}$, and therefore $P_{A} P_{B}=P_{B}=P_{B} P_{A}$.

At times, we will need to refer to Hamiltonians both in the bulk (2D) and at the boundary (1D). In order to avoid confusion, we will always denote one dimensional boundary Hamiltonians by the letters $Q, R, S, T$, while the parent Hamiltonian of the PEPS will always be referred to as $H$. 


\section{B. Boundary states of PEPS}

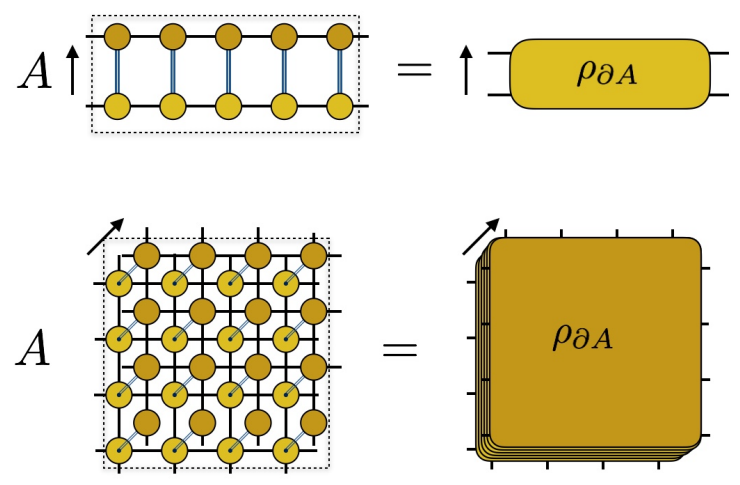

FIG. 2: Setup of the boundary state in one and two dimensions. The arrows indicate the input and output directions for the (unnormalized) boundary density matrix $\rho_{\partial A}$.

The main conceptual contribution of this paper is that 'boundary states' play a very important role in the analysis of ground state projectors for PEPS. These will be (unnormalized) positive operators acting on the virtual space associated with the edges connecting a region $A$ and its complement. They are obtained by contracting the physical indices inside $A$, and leaving the virtual indices at the boundary open, as depicted in Fig. 2

Definition 2. For a finite region $A \subseteq \Lambda$, the boundary state of $A$ is

$$
\rho_{\partial A}:=V_{A}^{\dagger} V_{A} \in \mathcal{B}\left(\mathcal{H}_{\partial A}\right) \text {. }
$$

Moreover, we define the following linear operator $W_{A}: \mathcal{H}_{\partial A} \rightarrow \mathcal{H}_{A}$

$$
W_{A}=V_{A} \rho_{\partial A}^{-1 / 2}
$$

where the inverse is taken on the support of $\rho_{\partial A}$ if it is not full rank.

Remark 3. Some properties of $\rho_{\partial A}$ and $W_{A}$ follow immediately from the definition

1. $\rho_{\partial A}$ is positive semi-definite;

2. $\operatorname{ker} \rho_{\partial A}=\operatorname{ker} V_{A}$, and in particular $\rho_{\partial A}>0$ if the PEPS is injective;

3. $W_{A} W_{A}^{\dagger}=V_{A} \rho_{\partial A}^{-1} V_{A}^{\dagger}=P_{A}$;

4. $W_{A}^{\dagger} W_{A}=\mathbb{1}_{\left(\operatorname{ker} V_{A}\right)^{\perp}}$, and therefore $W_{A}$ is a unitary from $\left(\operatorname{ker} V_{A}\right)^{\perp}$ to $\operatorname{Im} V_{A}$, and a partial isometry from $\mathcal{H}_{\partial A}$ to $\mathcal{H}_{A}$ (an isometry if the PEPS is injective).

The only point which might not be immediately clear from the definition is the fact that $P_{A}=W_{A} W_{A}^{\dagger}$ : this can be shown by observing that $W_{A} W_{A}^{\dagger}$ is a projector, which commutes with $P_{A}$ since $P_{A} V_{A}=V_{A}$, and has exactly the same image space as $V_{A}$ (and thus $P_{A}$ ).

Remark 4 . The entanglement spectrum is the spectrum of the reduced density matrix of a pure state [25]. In the case where $\Lambda$ has no outgoing edges, the entanglement spectrum is related to the boundary state in the following way: call $A^{c}=\Lambda \backslash A$ and note that

$$
\begin{aligned}
\operatorname{tr}_{A^{c}}\left[\left|\mathrm{PEPS}_{\Lambda}\right\rangle\left\langle\mathrm{PEPS}_{\Lambda}\right|\right] & =V_{A} \rho_{\partial A^{c}} V_{A}^{\dagger} \\
& =W_{A} \rho_{\partial A}^{1 / 2} \rho_{\partial A^{c}} \rho_{\partial A}^{1 / 2} W_{A}^{\dagger} .
\end{aligned}
$$


$W_{A}$ is an isometry, so the spectrum of the reduced state on $A$ is equal to the spectrum of $\rho_{\partial A}^{1 / 2} \rho_{\partial A^{c}} \rho_{\partial A}^{1 / 2}$. Here, $\rho_{\partial A}$ and $\rho_{\partial A^{c}}$ are different states that live on the same boundary $\partial A=\partial A^{c} . \rho_{\partial A}$ is constructed by contracting all of the physical indices in $A$, while $\rho_{\partial A}$ is constructed by contracting the physical indices outside of $A$. In certain, very special setups, these two states end up being equal, and so the entanglement spectrum, is equal to the spectrum of $\rho_{\partial A}^{2}$. This is the case in Ref. [26], where the system has periodic boundary conditions, and it is split exactly in two.

\section{The martingale condition and the spectral gap}

The main result of the paper is to show that under certain conditions, the parent Hamiltonian of a PEPS is gapped. In order to show this, we will invoke an equivalence theorem between the approximate commutativity of ground state projectors and the spectral gap of a frustration free Hamiltonian [33, 46]. This equivalence has adopted the name 'the martingale method' although its connection to martingales in probability theory is tenuous at best. We start by defining the spectral gap of a Hamiltonian $H$.

Definition 5. Let $\left\{H_{\Lambda}: H_{\Lambda} \in \mathcal{B}\left(\mathcal{H}_{\Lambda}\right)\right\}_{\Lambda}$ be a collection of Hamiltonians indexed by some set of regions $\Lambda \subset G$. The spectral gap of $H_{\Lambda}$, which we will denote by $\lambda(\Lambda)$, is defined as the difference between the two smallest distinct eigenvalues of $H_{\Lambda}$. We say that the family $\left\{H_{\Lambda}\right\}_{\Lambda}$ is gapped if

$$
\inf _{\Lambda} \lambda(\Lambda)>0
$$

It is clear that the condition of being gapped is non trivial only in the case of an infinite family of Hamiltonians, and in particular we are interested in showing that $\lambda(\Lambda)$ is lower bounded by a constant independent of the volume $|\Lambda|$ as $\Lambda$ tends to $G$. We will only consider families of Hamiltonians which are local (i.e. which can be constructed as sum of local interactions) and frustration-free (i.e. for which groundstates are the eigenvalues with minimal energy of all the local interaction terms).

Definition 6. Fix $r \in \mathbb{N}$. A Hamiltonian $H_{\Lambda}$ is said to be $r$-local if it can be decomposed as

$$
H_{\Lambda}=\sum_{Z \subset \Lambda} h_{Z}^{\Lambda},
$$

where each $h_{Z}^{\Lambda} \in \mathcal{B}\left(\mathcal{H}_{Z}\right)$ is Hermitian, and moreover $h_{Z}^{\Lambda}=0$ if $\operatorname{diam} Z>r$. The value $r$ will be called the range and $J=\sup _{Z}\left\|h_{Z}\right\|$ the strength of $H_{\Lambda}$. Moreover, a family $\left\{H_{\Lambda}\right\}_{\Lambda}$ of Hamiltonians will be said to be local if there is a choice of $r$ and $J$ such that $H_{\Lambda}$ is $r$-local with strength less than $J$, uniformly in $\Lambda$.

In a slight abuse of language, we will say that a (single) Hamiltonian $H_{\Lambda}$ is local (without specifying the range) if it belongs to a local family of Hamiltonians. We will also restrict to Hamiltonians where interactions terms are given as projectors, and which are frustration free. Assuming that the interactions are projections is not restrictive for finite range Hamiltonians, as long as each of the local terms $h_{Z}^{\Lambda}$ can be lower bounded by the projector on its range, up to a constant independent of $\Lambda$ and $Z$, since this will not change the low-energy properties of the Hamiltonian.

Definition 7 (Frustration free). Let $H_{\Lambda}=\sum_{Z} h_{Z}^{\Lambda}$ be a $r$-local Hamiltonian, and let $P_{\Lambda}$ be the projector on its groundstate space. We say that $H_{\Lambda}$ is frustration-free if $h_{Z}^{\Lambda}$ is an orthogonal projector and $h_{Z}^{\Lambda} P_{\Lambda}=0$ for all $Z$. 
In the case of frustration free Hamiltonians, the spectral gap is simply the smallest non-zero eigenvalue of the Hamiltonian.

We can now state the theorem relating ground state projectors to the spectral gap of $H_{\Lambda}$.

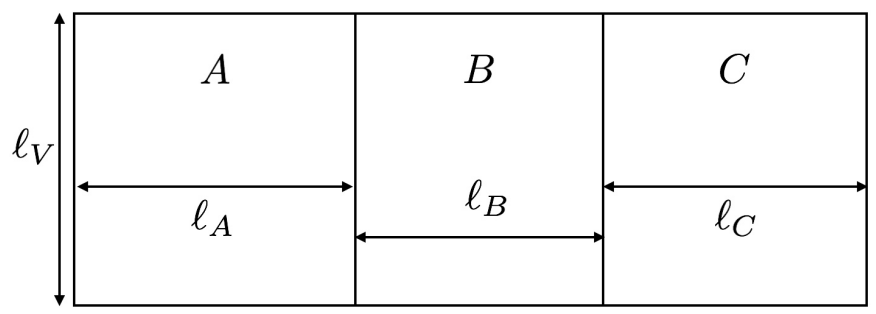

FIG. 3: Setup for the martingale condition in Theorem 8

Theorem 8 ([46]). Let $\left\{H_{\Lambda}\right\}_{\Lambda}$ be a family of local, frustration-free Hamiltonians defined on square regions $\Lambda \subset G$ (in $2 D$ ) whose local terms are orthogonal projections. If there exist positive constants $c$ and $\alpha$ and $\beta \in(0,1)$, and if for every three adjacent rectangles $A, B, C \subset \Lambda$ as depicted in Fig. 3. such that the width of $B$ separating $A$ and $C$ is $\ell_{B} \geq$ $\left(L^{*}\right)^{\beta}$ where $L^{*}=\max \left\{\ell_{V}, \ell_{A}, \ell_{C}\right\}$, the following holds

$$
\left\|P_{A B} P_{B C}-P_{A B C}\right\| \leq c \ell_{B}^{-\alpha},
$$

then $\lambda(\Lambda)$ is bounded from below by a constant independent of $|\Lambda|$. Conversely, if $\left\{H_{\Lambda}\right\}_{\Lambda}$ is gapped, then for every $\Lambda=A B C$

$$
\left\|P_{A B} P_{B C}-P_{A B C}\right\| \leq c e^{-\ell_{B} / \xi},
$$

for some constants $c, \xi$.

See Ref. [46] for a detailed proof and discussion of the theorem in any dimension. Eqn. (10) is referred to as the martingale condition. The norm on the r.h.s. of the equation can be understood as a strong measure of correlations in the groundstate. One way of seeing this is to observing that the expression is not small if there is a completely delocalized excitation: a state not in the groundstate of $A B C$, but which cannot be recognized as such by looking at regions $A B$ and $B C$ alone (so that the excitation is "hidden" and can only be measured if we have access to $A$ and $C$ at the same time). Theorem 8 then states that if this particular measure of correlations is decaying sufficiently fast in the overlap between regions, then the system is gapped and the decay is actually exponential. The usefulness of this characterization is due to the fact that, in the case of PEPS, a complete description of the groundstate subspace is available.

In the remainder of the paper, we will focus on proving that local boundary states imply the martingale condition, which will immediately imply that the parent Hamiltonian is gapped. An immediate corollary of Theorem 8 is that Eqn. (10) implies exponential decay of correlation in the ground state $\left|\Psi_{\Lambda}\right\rangle$ of $H_{\Lambda}$ if the ground state is unique:

$$
\left|\left\langle\Psi_{\Lambda}\left|f^{\dagger} g\right| \Psi_{\Lambda}\right\rangle-\left\langle\Psi_{\Lambda}\left|f^{\dagger}\right| \Psi_{\Lambda}\right\rangle\left\langle\Psi_{\Lambda}|g| \Psi_{\Lambda}\right\rangle\right| \leq \sqrt{\left\langle\Psi_{\Lambda}\left|f^{\dagger} f\right| \Psi_{\Lambda}\right\rangle\left\langle\Psi_{\Lambda}\left|g^{\dagger} g\right| \Psi_{\Lambda}\right\rangle} \gamma^{d(f, g)},
$$

where $d(f, g)$ is the lattice distance between the supports of operators $f, g$ and $\gamma \in(0,1)$ is some constant [53]. 


\section{THE GAP THEOREM FOR INJECTIVE PEPS}

\section{A. Approximate factorization of the boundary states}

In this section, we will give sufficient conditions on the boundary states such that the martingale condition (Eqn. (10) holds for any regions $A B C$ as in Fig. 3

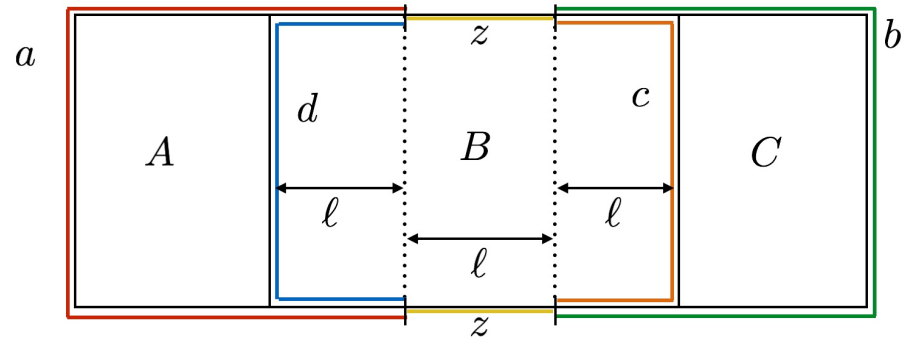

FIG. 4: Regions on the boundaries are labeled with lower case roman letters. Both $a, d$ and $c, d$ overlap on a region of length $\ell$, which is also a lower bound for the size of region $z$. All possible boundaries of $A, B, C$ can now be reconstructed in terms of $a, b, c, d, z$, e.g. $\partial A B=a \cup z \cup c \equiv a z c$.

We consider a specific set of rectangles $A B C$ and assume that the width of $B$ is at least $3 \ell$ for some reference length scale $\ell$. In Fig. 4, we label regions of the boundaries with lower case roman letters. It is understood that $a, d$ overlap on a region of length at least $\ell$, and the same holds true for $c, d$, while they are all disjoint from region $z$. The boundaries of regions $A, B, C$ can now be reconstructed in terms of $a, b, c, d, z$. For instance, $\partial A B=$ $a \cup z \cup c \equiv a z c$. Given the notation introduced above, we consider four invertible matrices $\Delta_{a z}, \Delta_{z b}, \Omega_{d z}, \Omega_{z c}$, and define a set of operators

$$
\begin{aligned}
\sigma_{\partial A B C} & :=\Delta_{z b} \Delta_{a z}, & \sigma_{\partial B} & :=\Omega_{z c} \Omega_{d z}, \\
\sigma_{\partial A B} & :=\Omega_{z c} \Delta_{a z}, & \sigma_{\partial B C} & :=\Delta_{z b} \Omega_{d z} .
\end{aligned}
$$

The operators $\sigma$ are understood as approximate (unnormalized) boundary states that can be factorized into two overlapping (possibly non-Hermitian) operators, in much the same way as Gibbs states of commuting local Hamiltonians ${ }^{1}$. All operators involved are invertible. It is important to note that by construction $\sigma_{\partial B C} \sigma_{\partial B}^{-1} \sigma_{\partial A B}=\sigma_{\partial A B C}$, and $\sigma_{\partial A B}^{-1} \sigma_{\partial B} \sigma_{\partial B C}^{-1}=$ $\sigma_{\partial A B C}^{-1}$. The support of each operator is indicated by their subscript according to the labels in Fig. 4

Definition 9. Let regions $A B C$ be as in Fig. 3, and let $\rho_{\partial A B}, \rho_{\partial B}, \rho_{\partial B C}, \rho_{\partial A B C}$ be the boundary states of regions $A B, B, B C$ and $A B C$ respectively. We say that the boundary states are $\epsilon$-approximately factorizable with respect to regions $A B C$, if there exist states $\left\{\sigma_{\partial A B}, \sigma_{\partial B}, \sigma_{\partial B C}, \sigma_{\partial A B C}\right\}$ with decomposition as in (13) such that the following condi-

\footnotetext{
${ }^{1}$ Note that in the case of isometric PEPS [54], in which the parent Hamiltonian is made of commuting terms, the boundary states are indeed Gibbs states of commuting interactions and have exactly the form (13). As expected, in this case $\epsilon=0$ in Theorem 10
} 
tions hold:

$$
\begin{aligned}
\left\|\rho_{\partial R}^{1 / 2} \sigma_{\partial R}^{-1} \rho_{\partial R}^{1 / 2}-\mathbb{1}\right\| & \leq \epsilon \quad \text { for } R \in\{A B C, A B, B C\} \\
\left\|\rho_{\partial B}^{-1 / 2} \sigma_{\partial B} \rho_{\partial B}^{-1 / 2}-\mathbb{1}\right\| & \leq \epsilon .
\end{aligned}
$$

We can now state the first main result of the paper:

Theorem 10. If the boundary states of regions $A B C$ are $\epsilon$-approximately factorizable for some $\epsilon \leq 1$, then $\left\|P_{A B} P_{B C}-P_{A B C}\right\| \leq 8 \epsilon$.

By Theorem 8 , we get that if for any sufficiently large regions $A B C$, where $B$ has diameter $\ell$, the boundary states are $\epsilon(\ell)$-approximately factorizable, for a sufficiently fast decaying $\epsilon(\ell)$, then the parent Hamiltonian of the PEPS is gapped.

In order to prove Theorem 10, we first start by showing that the measure of distance between $\rho_{\partial X}$ and $\sigma_{\partial X}$ (for $X$ being $A B, B C$, or $A B C$ ) is the "correct" one for the application we need.

Lemma 11. For a region $X \subset \Lambda$, let $\rho_{\partial X}$ be the boundary state and $\sigma_{\partial X}$ another invertible operator on $\mathcal{H}_{\partial X}$. Let $\tilde{P}_{X}=V_{X} \sigma_{\partial X}^{-1} V_{X}^{\dagger}$. Then

$$
\begin{aligned}
\left\|\tilde{P}_{X}\right\| & =\left\|\rho_{\partial X}^{1 / 2} \sigma_{\partial X}^{-1} \rho_{\partial X}^{1 / 2}\right\|, \\
\left\|P_{X}-\tilde{P}_{X}\right\| & =\left\|\rho_{\partial X}^{1 / 2} \sigma_{\partial X}^{-1} \rho_{\partial X}^{1 / 2}-\mathbb{1}\right\| .
\end{aligned}
$$

Proof. We first recall that $P_{X}=V_{X} \rho_{\partial X}^{-1} V_{X}^{\dagger}=W_{X} W_{X}^{\dagger}$. Therefore, we can rewrite $\tilde{P}_{X}$ as follows:

$$
\tilde{P}_{X}=V_{X} \sigma_{\partial X}^{-1} V_{X}^{\dagger}=V_{X} \rho_{X}^{-1 / 2}\left(\rho_{X}^{1 / 2} \sigma_{X}^{-1} \rho_{X}^{1 / 2}\right) \rho_{X}^{-1 / 2} V_{X}^{\dagger}=W_{X}\left(\rho_{X}^{1 / 2} \sigma_{X}^{-1} \rho_{X}^{1 / 2}\right) W_{X}^{\dagger} .
$$

Then Eq. (16) follow immediately from the fact that $W_{X}$ is an isometry, and similarly does Eq. (17):

$$
\left\|P_{X}-\tilde{P}_{X}\right\|=\left\|W_{X}\left(\mathbb{1}-\rho_{X}^{1 / 2} \sigma_{X}^{-1} \rho_{X}^{1 / 2}\right) W_{X}^{\dagger}\right\|=\left\|\mathbb{1}-\rho_{X}^{1 / 2} \sigma_{X}^{-1} \rho_{X}^{1 / 2}\right\| .
$$

We are now ready to prove Theorem 10

Proof of Theorem 10] A sketch of the proof is provided in Fig.5 for one dimensional systems, but provides the necessary intuition to follow the steps below. We start by setting some notation: given the regions $A$ and $B$, we denote by $\hat{V}_{A \rightarrow B}$ a modified version of $V_{A}$, where we have transposed from inputs to outputs the sites corresponding to the boundary shared between $A$ and $B$. $\hat{V}_{A \rightarrow B}$ then is a linear map from $\mathcal{H}_{\partial A \backslash \partial B}$ to $\mathcal{H}_{A} \otimes \mathcal{H}_{\partial A \cap \partial B}$, and we can write $V_{A B C}=V_{B C} \hat{V}_{A \rightarrow B}$ and $V_{A B}=V_{B} \hat{V}_{A \rightarrow B}$. We will do the same to define $\hat{V}_{C \rightarrow B}$. Using such notation, we see that

$\tilde{P}_{A B} \tilde{P}_{B C}=V_{A B} \sigma_{\partial A B}^{-1} \hat{V}_{A \rightarrow B}^{\dagger} V_{B}^{\dagger} V_{B} \hat{V}_{C \rightarrow B} \sigma_{\partial B C}^{-1} V_{B C}^{\dagger}=V_{A B} \sigma_{\partial A B}^{-1} \hat{V}_{A \rightarrow B}^{\dagger} \rho_{\partial B} \hat{V}_{C \rightarrow B} \sigma_{\partial B C}^{-1} V_{B C}^{\dagger}$,

while using (13) we have that:

$$
\begin{aligned}
\tilde{P}_{A B C} & =V_{A B C} \sigma_{\partial A B C}^{-1} V_{A B C}^{\dagger} \\
& =V_{A B} \hat{V}_{C \rightarrow B} \Delta_{a z}^{-1} \Delta_{z b}^{-1} \hat{V}_{A \rightarrow B}^{\dagger} V_{B C}^{\dagger} \\
& =V_{A B} \Delta_{a z}^{-1} \hat{V}_{A \rightarrow B}^{\dagger} \hat{V}_{C \rightarrow B}^{\dagger} \Delta_{z b}^{-1} V_{B C}^{\dagger} \\
& =V_{A B} \Delta_{a z}^{-1} \Omega_{z c}^{-1} \hat{V}_{A \rightarrow B}^{\dagger} \Omega_{z c} \Omega_{d z} \hat{V}_{C \rightarrow B}^{\dagger} \Omega_{d z}^{-1} \Delta_{z b}^{-1} V_{B C}^{\dagger} \\
& =V_{A B} \sigma_{\partial A B}^{-1} \hat{V}_{A \rightarrow B}^{\dagger} \sigma_{\partial B} \hat{V}_{C \rightarrow B} \sigma_{\partial B C}^{-1} V_{B C}^{\dagger} .
\end{aligned}
$$



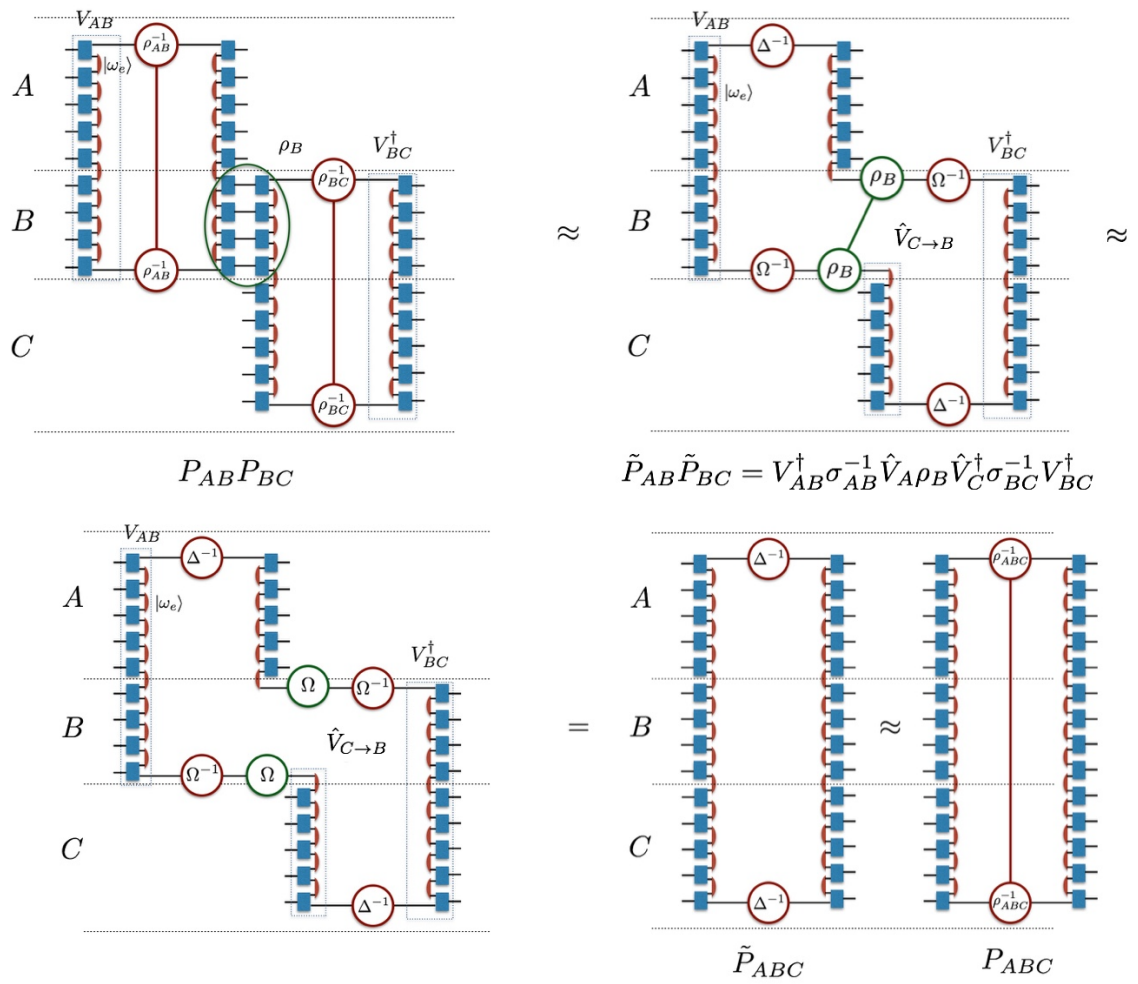

FIG. 5: Sketch of the proof in 1D. In the first step we simply write out the product of $P_{A B} P_{B C}$ in terms of the operators $V$ and the boundary states $\rho$. Then, using Eqn. 15, we approximate the boundary states of $A B$ and $B C$ by factorized operators $(\Omega, \Delta)$, giving us $\tilde{P}_{A B} \tilde{P}_{B C}$. In the next step we approximate the boundary state of $B$ by the factorized from Eqn. (14). Then the two ends of the respective boundaries cancel out, and we are left with $\tilde{P}_{A B C}$. In the final step we approximate the factorized boundary state on $A B C$ with the real boundary state using Eqn. 14 again.

We can therefore bound their difference as follows

$$
\begin{array}{r}
\left\|\tilde{P}_{A B} \tilde{P}_{B C}-\tilde{P}_{A B C}\right\|=\left\|V_{A B} \sigma_{\partial A B}^{-1} \hat{V}_{A \rightarrow B}^{\dagger}\left(\rho_{\partial B}-\sigma_{\partial B}\right) \hat{V}_{C \rightarrow B} \sigma_{\partial B C}^{-1} V_{B C}^{\dagger}\right\| \\
=\left\|V_{A B} \sigma_{\partial A B}^{-1} \hat{V}_{A \rightarrow B}^{\dagger} \rho_{\partial B}^{1 / 2}\left(\mathbb{1}-\rho_{\partial B}^{-1 / 2} \sigma_{\partial B} \rho_{\partial B}^{-1 / 2}\right) \rho_{\partial B}^{1 / 2} \hat{V}_{C \rightarrow B} \sigma_{\partial B C}^{-1}\right\| \\
\leq\left\|V_{A B} \sigma_{\partial A B}^{-1} \hat{V}_{A \rightarrow B}^{\dagger} \rho_{\partial B}^{1 / 2}\right\|\left\|V_{B C} \sigma_{\partial B C}^{-1} \hat{V}_{C \rightarrow B}^{\dagger} \rho_{\partial B}^{1 / 2}\right\|\left\|\mathbb{1}-\rho_{\partial B}^{-1 / 2} \sigma_{\partial B} \rho_{\partial B}^{-1 / 2}\right\| .
\end{array}
$$

In order to bound the first two terms in the r.h.s. of the last equation, we observe that

$$
\begin{aligned}
\left(V_{A B} \sigma_{\partial A B}^{-1} \hat{V}_{A \rightarrow B}^{\dagger} \rho_{\partial B}^{1 / 2}\right)\left(V_{A B} \sigma_{\partial A B}^{-1} \hat{V}_{A \rightarrow B}^{\dagger} \rho_{\partial B}^{1 / 2}\right)^{\dagger} & \\
& =V_{A B} \sigma_{\partial A B}^{-1} \hat{V}_{A \rightarrow B}^{\dagger} \rho_{\partial B} \hat{V}_{A \rightarrow B} \sigma_{\partial A B}^{-1} V_{A B}^{\dagger}=\tilde{P}_{A B}^{2},
\end{aligned}
$$

which implies that

$$
\left\|V_{A B} \sigma_{\partial A B}^{-1} \hat{V}_{A \rightarrow B}^{\dagger} \rho_{\partial B}^{1 / 2}\right\|=\left\|\tilde{P}_{A B}^{2}\right\|^{1 / 2} \leq\left\|\tilde{P}_{A B}\right\| .
$$


Since the same holds for the terms on $B C$, we have proven that

$$
\left\|\tilde{P}_{A B} \tilde{P}_{B C}-\tilde{P}_{A B C}\right\| \leq\left\|\tilde{P}_{A B}\right\|\left\|\tilde{P}_{B C}\right\|\left\|\mathbb{1}-\rho_{\partial B}^{-1 / 2} \sigma_{\partial B} \rho_{\partial B}^{-1 / 2}\right\| .
$$

We can now apply Lemma 11 and Eqs. (14) and (15), to get

$$
\left\|\tilde{P}_{A B} \tilde{P}_{B C}-\tilde{P}_{A B C}\right\| \leq(1+\epsilon)^{2} \epsilon
$$

since $\left\|\tilde{P}_{A B}\right\| \leq\left\|\tilde{P}_{A B}-P_{A B}\right\|+\left\|P_{A B}\right\| \leq \epsilon+1$.

To conclude the proof, we just have to resort one last time to Lemma 11, as we have that:

$$
\begin{aligned}
\left\|P_{A B} P_{B C}-P_{A B C}\right\| \leq & \left\|\tilde{P}_{A B} \tilde{P}_{B C}-\tilde{P}_{A B C}\right\|+\left\|\tilde{P}_{A B}\right\|\left\|P_{B C}-\tilde{P}_{B C}\right\| \\
& +\left\|P_{A B}-\tilde{P}_{A B}\right\|\left\|P_{B C}\right\|+\left\|\tilde{P}_{A B C}-P_{A B C}\right\| \\
\leq & (1+\epsilon)^{2} \epsilon+(1+\epsilon) \epsilon+2 \epsilon \leq 8 \epsilon .
\end{aligned}
$$

\section{B. Matrix Product States}

As is often the case, in one dimension the situation becomes particularly simple since the boundary is zero dimensional and has two spatially separated ends. We will show that the boundary state of an injective MPS is approximately factorizable when the region is long enough. Consider a translationally invariant MPS on a chain of length $N$ :

$$
|\mathrm{MPS}\rangle=\sum_{j_{1}, \cdots, j_{N}=1}^{d} \operatorname{tr}\left\{T_{j_{1}} \cdots T_{j_{N}}\right\}\left|j_{1}, \cdots, j_{N}\right\rangle,
$$

where $\left\{T_{j}\right\}_{j=1}^{d}$ is a collection of $d$ matrices of size $D \times D$.

Many of the properties of an MPS can be succinctly described by the transfer operator $\mathbb{E}(f)=\sum_{j} T_{j} f T_{j}^{\dagger}$, which maps virtual bonds to virtual bonds by contracting one single physical bond (see Fig. 6). The boundary state $\rho_{\partial A}$ of a region $A$ of length $m$ is the Choi matrix of the $m$-th power of $\mathbb{E}$ :

$$
\rho_{\partial A}=\mathbb{E}^{m} \otimes \operatorname{id}(|\Omega\rangle\langle\Omega|), \quad|\Omega\rangle=\sum_{i=1}^{D}|i, i\rangle .
$$

Note that by construction $\mathbb{E}$ is a completely positive map, so its Choi matrix is a positive operator.

If we replace the matrices $T_{j}$ with matrices $Z T_{j} Z^{-1}$, for some invertible matrix $Z$, it is easy to see that the state $|\mathrm{MPS}\rangle$ is left invariant [54] (although the boundary state $\rho_{\partial A}$ will not: it will be mapped to $\left(Z \otimes Z^{-1}\right)^{\dagger} \rho_{\partial A}\left(Z \otimes Z^{-1}\right)$ ). This operation is usually referred to as "choosing the gauge" of the MPS representation. It is a well known fact that, in the case of injective MPS, there is a choice of gauge that makes the transfer operator a trace-preserving map [54]. This allows us to prove the following property of the boundary states of an injective MPS. 

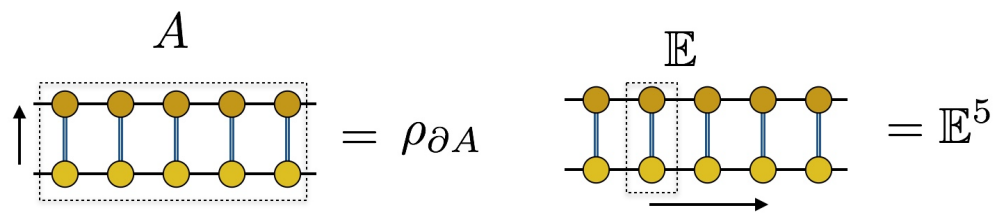

FIG. 6: In one dimension, the boundary state can be reinterpreted as a power of the transfer operator when we flip the indices from reading up-down to left-right.

Lemma 12. Given an injective MPS, there is a representation with the following property: there exist a full rank state $\sigma \in \mathcal{B}\left(\mathbb{C}^{d}\right)$, a positive constant $c$ and $a \gamma \in(0,1)$ such that, for every chain A of length $m$ it holds that

$$
\left\|\rho_{\partial A}-\sigma \otimes \mathbb{1}\right\|_{1} \leq c \gamma^{m},
$$

where $\rho_{\partial A}$ is the boundary state on $A$.

Proof. The MPS is injective on a chain of length $m$ if and only if the set $\left\{T_{j_{1}} \cdots T_{j_{m}} \mid j_{1}, \ldots, j_{m}=1 \ldots d\right\}$ spans the full matrix algebra $\mathcal{B}\left(\mathbb{C}^{D}\right)$ : but since that is exactly the set of Kraus operator of $\mathbb{E}^{m}$, it implies that $\mathbb{E}$ is primitive (a primitive linear map is an irreducible positive map with trivial peripheral spectrum) [55]. The adjoint map $\mathbb{E}^{*}(g)=\sum_{j} T_{j}^{\dagger} g T_{j}$ will share the same property, so that we can find a positive-definite operator $y$ such that $\mathbb{E}^{*}(y)=\lambda y$ where $\lambda>0$ is the largest eigenvalue of $\mathbb{E}$, which is also simple. We can then change the matrices in the MPS representation, replacing each $T_{j}$ with $\frac{1}{\lambda} y^{1 / 2} T_{j} y^{-1 / 2}$, without changing the state $|\mathrm{MPS}\rangle$ (apart from its normalization). With this choice of gauge, the transfer operator is trace preserving, since $\mathbb{E}^{*}(\mathbb{1})=\mathbb{1}$. Moreover, the sequence of linear maps $\mathbb{E}^{m}$ will converge, as $m$ goes to infinity, to another completely positive, trace preserving map $\mathbb{E}^{\infty}$, which is of the form $\mathbb{E}^{\infty}(f)=\operatorname{tr}\{f\} \sigma$, for some fixed $\sigma>0$. The Choi matrix of $\mathbb{E}^{\infty}$ is given by

$$
\begin{aligned}
\tau_{\mathbb{E} \infty} & =\lim _{m \rightarrow \infty} \mathbb{E}^{m} \otimes \operatorname{id}(|\Omega\rangle\langle\Omega|) \\
& =\sum_{j k} \lim _{m \rightarrow \infty} \mathbb{E}^{m}(|j\rangle\langle k|) \otimes|j\rangle\langle k| \\
& =\sum_{j} \sigma \otimes|j\rangle\langle j|=\sigma \otimes \mathbb{1} .
\end{aligned}
$$

Since the dimension $D$ is fixed, by the Jordan decomposition there exists some $\gamma \in(0,1)$ and some constant $c>0$ such that

$$
\left\|\mathbb{E}^{m}-\mathbb{E}^{\infty}\right\|_{1 \rightarrow 1, c b} \leq c \gamma^{m},
$$

where $\|\cdot\|_{1 \rightarrow 1, c b}$ is the completely bounded $1 \rightarrow 1$ norm, also known as the diamond norm. Furthermore, the difference between the Choi matrix of two channels is bounded by their difference in diamond norm $\left\|\tau_{T}-\tau_{S}\right\|_{1} \leq\|T-S\|_{1 \rightarrow 1, c b}$. This is obvious since $\|T-S\|_{1 \rightarrow 1, c b}=\sup _{\rho}\|T \otimes \operatorname{id}(\rho)-S \otimes \operatorname{id}(\rho)\|_{1}$. By this argument, we get that for this specific choice of gauge, if $A$ is a chain of length $m$, it holds that

$$
\left\|\rho_{\partial A}-\sigma \otimes \mathbb{1}\right\|_{1} \leq c \gamma^{m} .
$$


Since we are working in finite dimension (every $\rho_{\partial A}$ lives in the same space $\mathcal{B}\left(\mathbb{C}^{D}\right)$ independently of $m$ ), the fact that $\rho_{\partial A}$ converges in the trace norm to a product state is sufficient to prove that is approximately factorizable in the sense of equations (14) and (15). To show this, we first need the following lemma.

Lemma 13. If $X, Y$ are positive operators, and $Y$ is invertible, it holds that:

$$
\left\|X^{1 / 2} Y^{-1} X^{1 / 2}-\mathbb{1}\right\| \leq Y_{\min }^{-1}\|X-Y\|
$$

where $Y_{\min }$ is the smallest eigenvalue of $Y$.

Proof. First of all, we rewrite $X^{1 / 2} Y^{-1} X^{1 / 2}-\mathbb{1}$ as $X^{1 / 2}\left(Y^{-1}-X^{-1}\right) X^{1 / 2}$, where the inverse of $X$ is taken on its support. $X^{1 / 2}\left(Y^{-1}-X^{-1}\right) X^{1 / 2}$ is a normal operator with the same spectrum as $\left(Y^{-1}-X^{-1}\right) X$. Since the spectral radius of an operator is a lower bound to its operator norm, and for normal operators equality holds, we have that:

$$
\left\|X^{1 / 2}\left(Y^{-1}-X^{-1}\right) X^{1 / 2}\right\| \leq\left\|\left(Y^{-1}-X^{-1}\right) X\right\| .
$$

(See also [56, Proposition IX.1.1]). We can now rewrite $\left(Y^{-1}-X^{-1}\right) X$ as $Y^{-1}(X-Y)$, so that we obtain

$$
\left\|X^{1 / 2} Y^{-1} X^{1 / 2}-\mathbb{1}\right\| \leq\left\|Y^{-1}(X-Y)\right\| \leq Y_{\min }^{-1}\|X-Y\| .
$$

To conclude that $\rho_{\partial A}$ is approximately factorizable, we can then apply Lemma 13 to $\rho_{\partial A}$ and $\sigma \otimes \mathbb{1}$, and we obtain that

$$
\left\|\rho_{\partial A}^{1 / 2}\left(\sigma^{-1} \otimes \mathbb{1}\right) \rho_{\partial A}^{1 / 2}-\mathbb{1}\right\| \leq \sigma_{\min }^{-1}\left\|\rho_{\partial A}-\sigma \otimes \mathbb{1}\right\|_{1} \leq c \sigma_{\min }^{-1} \gamma^{m},
$$

since we can upper bound the operator norm with the trace norm. Therefore, it will decay exponentially in the length of $A$. We can more easily bound $\left\|\rho_{\partial A}^{-1 / 2}\left(\sigma^{1 / 2} \otimes \mathbb{1}\right) \rho_{\partial A}^{-1 / 2}-\mathbb{1}\right\|$ by $r_{\partial A}^{-1}\left\|\rho_{\partial A}-\sigma\right\|_{1}$, where $r_{\partial A}$ is the minimal eigenvalue of $\rho_{\partial A}$. We can then observe that $r_{\partial A}$ is lower bounded by $\sigma_{\min }-\left\|\rho_{\partial A}-\sigma\right\|$. Therefore, if $m$ is sufficiently large, we can assume that $r_{\partial A}$ is larger than $\sigma_{\min } / 2$, so that we also have

$$
\left\|\rho_{\partial A}^{-1 / 2}\left(\sigma^{1 / 2} \otimes \mathbb{1}\right) \rho_{\partial A}^{-1 / 2}-\mathbb{1}\right\| \leq c^{\prime} \sigma_{\min }^{-1} \gamma^{m}
$$

for some positive constant $c^{\prime}$.

\section{THE GAP THEOREM FOR NON-INJECTIVE PEPS}

All of the results so far have been obtained for injective PEPS. Injectivity is essential in guaranteeing that the boundary state $\rho_{\partial X}$ is full rank and hence invertible. In the MPS setting, we also saw that injectivity is sufficient to show approximate factorization. However, injective PEPS exclude any description of topologically ordered phases. In this section we consider extensions of injectivity (G-injectivity and MPO-injectivity) that allow for the description of most known topological phases of gapped spin systems. We show that with a slight modification of definitions, we can extend Theorem 10 to the setting of MPO-injectivity. 


\section{A. MPO-injectivity}

Injective PEPS can be seen as perturbations of trivial short-range entangled states (products of nearest-neighbor maximally entangled states) via Eqn. (3). In the same way, more complicated states, such as the toric code or other topologically-ordered states, can be taken as base states to be perturbed, giving rise to different classes of PEPS.

A first construction is to take as base state |Base $\rangle$ the so called $G$-isometric PEPS [14], for a given finite group $G$. They correspond to Kitaev's quantum double models $D(G)$. In particular for the group $G=\mathbb{Z}_{2}, \mid$ Base $\rangle$ is just the toric code. $G$-isometric PEPS are defined by fixing the bond and physical dimensions respectively to $D=|G|, d=D^{\otimes 4}$ and by choosing as PEPS tensor $T_{v}=\frac{1}{|G|} \sum_{g \in G} L_{g}^{\otimes 4}$ in Eqn. 3, with $L_{g}$ being the left regular representation of $G$.

It was shown in [17] that the parent Hamiltonian of a $G$-isometric PEPS is commuting, and that the boundary state $\rho_{\partial A}$ of the PEPS in a region $A \subset \Lambda$, as defined previously in the text (Figure 2), is exactly the projector $J_{\partial A}:=\frac{1}{|G|} \sum_{g \in G} L_{g}^{\otimes|\partial A|}$. Note that $J_{\partial A}$ can be written as a translational invariant Matrix Product Operator (MPO) with bond dimension $|G|$ :

$$
J_{N_{\text {sites }}}=\frac{1}{|G|} \sum_{g_{1}, \ldots g_{N} \in G} \operatorname{tr}\left(B_{g_{1}} \cdots B_{g_{N}}\right) L_{g_{1}} \otimes \cdots \otimes L_{g_{N}} .
$$

For that, it is enough to take $B_{g}=|g\rangle\langle g|$.

Perturbed PEPS of the form $\bigotimes_{v \in \Lambda} Y_{v} \mid$ Base $\left._{\Lambda}\right\rangle$, with $Y_{v}$ invertible, where $\mid$ Base $\left._{\Lambda}\right\rangle$ is a $G$ isometric PEPS are called $G$-injective. The construction of $G$-injective PEPS was generalized first by Buerschaper in [15] and later by Sahinoglu et al in [16] considering as initial base state all Levin-Wen string-net states [41], which are believed to cover all possible 2D non-chiral topological phases. The starting point of the construction by Sahinoglu et al in [16] (see [18]) is a translational invariant MPO $J_{N}$ which is a projector for all system size $N$. As shown in [16, 18], by invoking the fundamental theorem of Matrix Product Vectors [17], this induces an algebra of MPO which in turn gives rise to a fusion category. The state $\mid$ Base $\left._{\Lambda}\right\rangle$ is defined in the same way as for the $G$-injective case: $T_{v}$ is given by the MPO-projector acting on four sites $J_{4}$. The resulting state $\bigotimes_{v \in \Lambda} Y_{v} \mid$ Base $\left._{\Lambda}\right\rangle$ is called an MPO-injective PEPS.

\section{B. Approximate factorization for MPO-injective PEPS}

Some simple properties of MPO-injective PEPS will be sufficient to extend the results of Theorem 10

Definition 14. Given a non-injective PEPS, for each region $A \subset \Lambda$ denote by $J_{\partial A} \in \mathcal{B}\left(\mathcal{H}_{\partial A}\right)$ the projector on the complement of the kernel of $\rho_{\partial A}$.

Note that the kernel of $\rho_{\partial A}$ coincides with the kernel of $V_{A}$ (see Remark 3), so that we trivially have $V_{A}=V_{A} J_{\partial A}$. We need a compatibility condition between the $J_{\partial A}$ acting on overlapping regions. We will use the modified map $\hat{V}_{A \rightarrow B}$ defined in the proof of Theorem 10.

Definition 15 (Pulling-through condition). A PEPS satisfies the pulling-through condition [16] if for every pair of contiguous regions $A$ and $B$, it holds that

$$
J_{\partial B} \hat{V}_{A \rightarrow B}=\hat{V}_{A \rightarrow B} J_{\partial A B}
$$




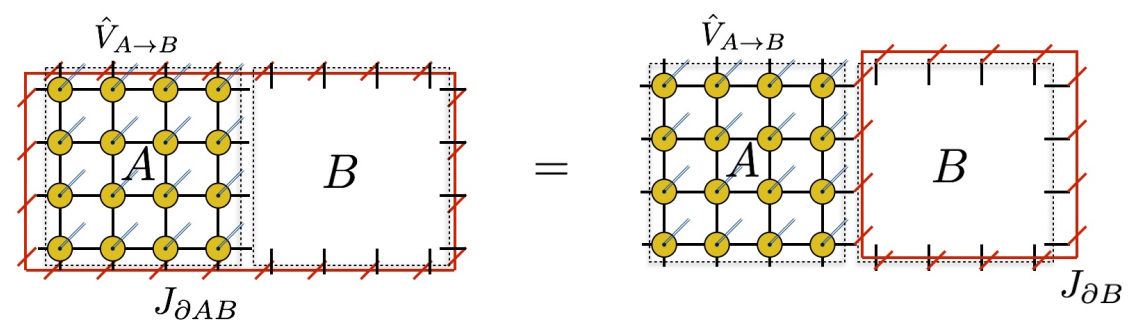

FIG. 7: Setup for pulling through condition. The MPO projector $J_{\partial B}$ is indicated in red, and acts on the boundary of $A B$ from the right (bottom in figure), and acts on the boundary of $B$ from the left (top in the figure).

As in Theorem 10, it is understood that the virtual indices of $\hat{V}_{A \rightarrow B}$ that touch $B$ are output variables rather than input variables as in $V_{A}$ (see Fig. 7).

We will now show that the proof of Theorem 10 can be adapted to the case of MPOinjective PEPS if we restrict all the operators living on the boundary to have support on the range of the projectors $J_{\partial A}$.

Definition 16. Let $A B C$ be regions as in Fig. 3 and $\rho_{\partial A B}, \rho_{\partial B C}, \rho_{\partial B}$ and $\rho_{\partial A B C}$ the boundary states of regions $A B, B C, B$ and $A B C$ respectively. We will say that the boundary states are $\epsilon$-approximately factorizable with respect to regions $A B C$, if there exist invertible operators $\Delta_{z b}, \Delta_{a z}, \Omega_{z c}, \Omega_{d z}$ with support given by Fig. 4, defining operators $\sigma_{\partial A B C}, \sigma_{\partial B}$, $\sigma_{\partial A B}$ and $\sigma_{\partial B C}$ as in (13), and the following holds:

$$
\begin{aligned}
{\left[J_{\partial R}, \sigma_{\partial R}\right] } & =0 \\
\left\|\rho_{\partial R}^{1 / 2} \sigma_{\partial R}^{-1} \rho_{\partial R}^{1 / 2}-J_{\partial R}\right\| & \leq \epsilon \quad \text { for } R \in\{A B C, A B, B C\} ; \\
\left\|\rho_{\partial B}^{-1 / 2} J_{\partial B} \sigma_{\partial B} J_{\partial B} \rho_{\partial B}^{-1 / 2}-J_{\partial B}\right\| & \leq \epsilon
\end{aligned}
$$

The reason for the change in Eqn. (25) compared to Eqns. (14) and (15) is clear given the following extension of Lemma 11 .

Lemma 17. For a region $X \subset \Lambda$, let $\rho_{\partial X}$ be the boundary state and $\sigma_{\partial X}$ another operator invertible on $J_{\partial X} \mathcal{H}_{\partial X} J_{\partial X}$. Let $\tilde{P}_{X}=V_{X} \sigma_{\partial X}^{-1} V_{X}^{\dagger}$. Then

$$
\begin{aligned}
\left\|\tilde{P}_{X}\right\| & =\left\|\rho_{\partial X}^{1 / 2} \sigma_{\partial X}^{-1} \rho_{\partial X}^{1 / 2}\right\|, \\
\left\|\tilde{P}_{X}-P_{X}\right\| & =\left\|\rho_{\partial X}^{1 / 2} \sigma_{\partial X}^{-1} \rho_{\partial X}^{1 / 2}-J_{\partial X}\right\| .
\end{aligned}
$$

Since the proof is identical to that of Lemma 11 , with the only difference that now $W_{X}$ is a partial isometry and $W_{X} W_{X}^{\dagger}=J_{\partial X}$, so we will omit it. By using this last lemma instead of Lemma 11] in the proof of Theorem 10, the proof carries through almost identically: the pulling-through condition guarantees that the projections $J_{\partial X}$ can be moved through the 
equation as needed. In particular, we have that

$$
\begin{aligned}
\tilde{P}_{A B C} & =V_{A B C} \sigma_{\partial A B C}^{-1} V_{A B C}^{\dagger} \\
& =V_{A B} \hat{V}_{C \rightarrow B} J_{\partial A B C} \Delta_{a z}^{-1} \Delta_{z b}^{-1} J_{\partial A B C} \hat{V}_{A \rightarrow B}^{\dagger} V_{B C}^{\dagger} \\
& =V_{A B} J_{\partial A B} \Delta_{a z}^{-1} \hat{V}_{A \rightarrow B}^{\dagger} \hat{V}_{C \rightarrow B} \Delta_{z b}^{-1} J_{\partial B C} V_{B C}^{\dagger} \\
& =V_{A B} J_{\partial A B} \Delta_{a z}^{-1} \Omega_{z c}^{-1} \hat{V}_{A \rightarrow B}^{\dagger} \Omega_{z c} \Omega_{d z} \hat{V}_{C \rightarrow B} \Omega_{d z}^{-1} \Delta z b^{-1} J_{\partial B C} V_{B C}^{\dagger} \\
& =V_{A B} J_{\partial A B} \sigma_{\partial A B}^{-1} \hat{V}_{A \rightarrow B}^{\dagger} \sigma_{\partial B} \hat{V}_{C \rightarrow B} \sigma_{\partial B C}^{-1} J_{\partial B C} V_{B C}^{\dagger} \\
& =V_{A B} \sigma_{\partial A B}^{-1} \hat{V}_{A \rightarrow B}^{\dagger} J_{\partial B} \sigma_{\partial B} J_{\partial B} \hat{V}_{C \rightarrow B} \sigma_{\partial B C}^{-1} V_{B C}^{\dagger} .
\end{aligned}
$$

The rest of the proof is identical to the non-topological case.

\section{APPROXIMATE FACTORIZATION OF 1D THERMAL STATES}

In this section, we consider a class of physically motivated states for which we can show that they are approximately factorizable, by explicitly constructing operators $\{\Delta, \Omega\}$ satisfying Eqns. 14, 15.

\section{A. Local and quasi-local Hamiltonians}

We define the following properties for families of operators $f_{\partial A}$ defined for every rectangular subset $A$ of the lattice, and acting on the boundary Hilbert space $\mathcal{H}_{\partial A}$.

Definition 18. Let $\left\{f_{\partial A}\right\}_{A}$ be a family of operators such that $f_{\partial A} \in \mathcal{B}\left(\mathcal{H}_{\partial A}\right)$, where the index $A$ runs over all rectangles $A \subset G$. For each $A$, we decompose $f_{\partial A}$ as follows:

$$
f_{\partial A}=\sum_{Z \subset \partial A} f_{Z}^{\partial A}
$$

where each $f_{Z}^{\partial A}$ is supported on $Z$ (such decomposition is always trivially possible). Moreover, if every $f_{\partial A}$ is Hermitian, we require every $f_{Z}^{\partial A}$ to be Hermitian as well. We will then say that:

locality: the family $\left\{f_{\partial A}\right\}_{A}$ is local, if there exist an integer $k^{*}$ and a constant $J>0$ such that

$$
\sup _{\partial A} \sup _{Z}\left\|f_{Z}^{\partial A}\right\| \leq J
$$

and moreover $f_{Z}=0$ if the diameter of $Z$ is larger than $k^{*}$. The value $k^{*}$ will denote the range of $f_{\partial A}$, while $J$ will be the strength of $f_{\partial A}$. We will also say that $f_{\partial A}$ is $k^{*}$-local.

quasi-locality: the family $\left\{f_{\partial A}\right\}_{A}$ is quasi-local, if

$$
\forall x \geq 1, \quad \sup _{A} \sup _{u \in \partial A} \sum_{Z \ni u} x^{\operatorname{diam} Z}\left\|f_{Z}^{\partial A}\right\|<\infty .
$$

The definition of quasi-locality implies that, for each $\partial A$, the norm $\left\|f_{Z}^{\partial_{A}}\right\|$ decays in the diameter of $Z$ faster than any exponential. This is quite a stronger requirement than what 
is usually made. In the setting of local Hamiltonians, one usually considers the case of exponential or faster than polynomial decaying interactions, while the quasi-local algebra for quantum spin systems in the thermodynamic limit usually contains any norm-convergent sequence of local operators [57]. The motivation for our choice of a stronger notion will be clear in the next section.

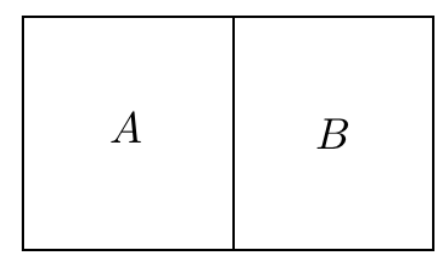

FIG. 8: Setup for the homogeneity assumption.

The next assumption relates the local terms of different (overlapping) regions: we require that different regions have approximately the same local terms over the segments where they overlap.

Definition 19 ( $\delta$-homogeneity). Let $\left\{f_{\partial A}\right\}_{A}$ be a family of quasi-local operators. We say that the family is $\delta$-homogeneous if for every pair of rectangles $A$ and $B$ arranged as in Fig. 8 and for every $Z \subset \partial A \backslash \partial B$,

$$
\left\|f_{Z}^{\partial A}-f_{Z}^{\partial A B}\right\| \leq \delta^{A B}(\operatorname{dist}(Z, \partial B)),
$$

for some family $\left\{\delta^{A}(r)\right\}_{A}$ of decaying functions. If there exists a constant $r^{*}$ for which $\delta^{A}(r)=0$ for every $r>r^{*}$ and every $A$, we say that the family $\left\{f_{\partial A}\right\}_{A}$ is strictly homogeneous.

It is quite clear that strict homogeneity only makes sense in the case of strict locality.

In the following result, we will consider regions $A B C \subseteq \Lambda$ as in Fig. 3 , and we will furthermore subdivide region $z$ of $\partial B$ into two parts, which we denote $x$ and $y$, as in Fig. 9 . We will assume that $B$ is $4 \ell$ thick, for some $\ell>0$, that moreover both region $x$ and $y$ are $\ell$ long.

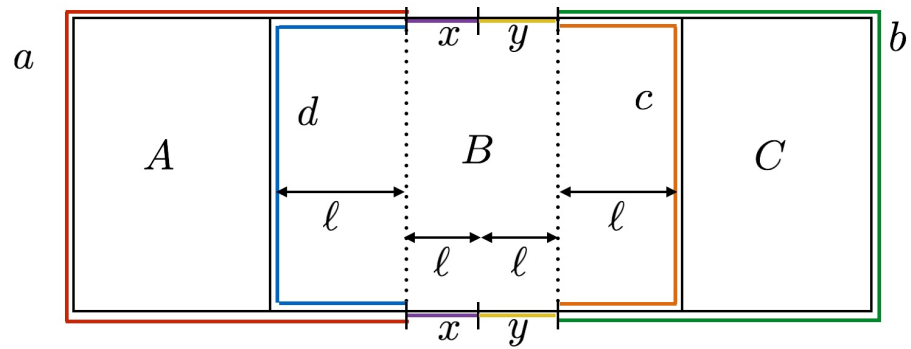

FIG. 9: We further decompose region $z$ into $x$ and $y$ as compared with Fig. 4

Theorem 20. Let us consider a family $\left\{Q_{\partial A}\right\}_{A \subset G}$ of local Hermitian operators with range $r$ and strength $J$, which is $\delta$-homogeneous, and let $\rho_{\partial A}=\exp \left(2 Q_{\partial A}\right)$ the corresponding thermal state for each $A \subset G$ (where the factor of 2 is added for convenience). Then there 
exists an $\ell_{0}$ such that for a rectangular region $\Lambda$ satisfying the conditions described in Fig. 3 with $\ell \geq \ell_{0}, \rho_{\partial \Lambda}$ is $\epsilon(\ell)$-approximately factorizable (equations (14) and (15)), where

$$
\epsilon(\ell)=\eta\left(c(r, J) \max \left\{\chi_{\ell}(2 J), \sum_{l \geq \ell} \delta^{\Lambda}(l)\right\}\right),
$$

where $\eta(t)=e^{t}-1, c(r, J)$ depends only on the range $r$ and the strength $J$, and $\chi_{\ell}(2 J)$ decays faster than any exponential in $\ell$.

\section{B. Analysis of 1D Gibbs states}

As a preliminary to the proof of Theorem 20, we will first recall some results about 1D local Hamiltonians, and some useful theory on expansionals. A very important set of tools in our analysis of thermal states has been developed by H. Araki [1] nearly a half a century ago, with the purpose of proving the absence of phase transitions at finite temperatures for 1D local Hamiltonians. On the way to proving that result, he also showed that the evolution associated to 1D local Hamiltonians is entire analytic, and that local observables preserve their locality, up to small errors, under imaginary time evolution.

Here we state the results of Araki in a language that suits us, and refer back to the original paper for the proofs. We will make use of the following notation, for every pair of operators $A$ and $B$ :

$$
\Gamma_{B}^{t}(A)=e^{t B} A e^{-t B} .
$$

Note that if $[A, B]=0$ then $\Gamma_{B}^{t}(A)=A$.

Consider the one dimensional local Hamiltonian $Q_{\Sigma}=\sum_{Z \subset \Sigma} q_{Z}$, where $\Sigma \subset \mathbb{Z}$ is a finite interval containing the origin, and the Hamiltonian terms $q_{Z}$ are zero if the radius of $Z$ is greater than $r$. For every integer $\ell$ let $Q_{[-\ell ; \ell]}=\sum_{Z \subset[-\ell ; \ell]} q_{Z}$ be the restriction to region $[-\ell ; \ell] \subset \Sigma$; i.e. all local Hamiltonian terms that are strictly inside of $[-\ell ; \ell]$. We will use the shorthand notation $\Gamma_{\Sigma}^{t}$ for $\Gamma_{Q_{\Sigma}}^{t}$ and $\Gamma_{\ell}^{t}$ for $\Gamma_{Q_{[-\ell, \ell]}}^{t}$ for the rest of this section.

We can now state Arakis' theorem:

Theorem 21 (Araki [1]). Let $Q_{\Sigma}=\sum_{Z \subseteq \Sigma} q_{Z}$ be a local Hamiltonian on $\Sigma$ with interaction length $r$ and strength $J$, and let $f$ be an observable with support on $[-n ; n]$. Then

$$
\begin{aligned}
\left\|\Gamma_{\Sigma}^{t}(f)-\Gamma_{\ell}^{t}(f)\right\| & \leq \chi_{\ell}(\tau) F_{n}(\tau)\|f\| \\
\left\|\Gamma_{\Sigma}^{t}(f)\right\| & \leq F_{n}(\tau)\|f\|,
\end{aligned}
$$

where $\tau=2 t J$ and the functions $\chi_{\ell}$ and $F_{n}$ can be bounded as

$$
F_{n}(x) \leq e^{(n-r+1) x+2 \log (r) e^{x r}}, \quad \text { and } \quad \chi_{\ell}(x) \leq \frac{\left[2 \log (r) e^{r x}\right]^{\lfloor/ r\rfloor+1}}{(\lfloor\ell / r\rfloor+1) !} .
$$

The bounds in Eqn. (36) only strictly holds for $x>c$ for some constant $c$ of order one. For very small $t$, the bound on $F_{n}(x)$ and $\chi_{\ell}(x)$ take on a slightly different functional form, that is mostly irrelevant for us now. The important thing to notice is that for any fixed $n$ and $x$, $F_{n}(x)$ is bounded, and $\chi_{\ell}(x)$ is decaying faster than any exponential in $\ell$, both uniformly in the size of $\Sigma$.

Araki's Theorem tells us that the imaginary time evolution of a local observable on a 1D line gets mapped to a quasi-local observable with the same center for every time $t$, which can 
be well approximated by evolution generated by restricted Hamiltonians. Although for real time evolution, this statement holds in lattices of any dimension, and is more widely known as a Lieb-Robison bound [58], it breaks down in 2 and higher dimensions for imaginary time. In fact, Araki's Theorem shows that it is possible to extend the time evolution $f \rightarrow$ $e^{i t Q_{\Sigma}} f e^{-i t Q_{\Sigma}}$ in the limit where $\Sigma \rightarrow \mathbb{Z}$, to an entire analytic function. This will not be true in higher dimensions, and the resulting evolution will only be analytic in a strip centered on the real line. An explicit counter-example was constructed in Ref. [59].

Note that, contrary to the Lieb-Robinson bound (which holds for real time evolution), where the support of the evolved observable grows linearly with time, Araki's Theorem can only bound the growth of the support with an exponential in time. Therefore the proof would not carry trough if we defined quasi-local observables to have exponentially decaying tails.

We finally point out an easy corollary of Theorem 21 that we will need in Section VD.

Corollary 22 (Araki [1]). Let $Q_{\Sigma}=\sum_{Z \subseteq \Sigma} q_{Z}$ be a local Hamiltonian on $\Sigma$ with interaction length $r$ and strength $J$, and let $f$ be a quasi-local observable with center at the origin. Then

$$
\begin{aligned}
\left\|\Gamma_{\Sigma}^{t}(f)-\Gamma_{\ell}^{t}(f)\right\| & \leq \chi_{\ell}(\tau) G(\tau)\|f\| \\
\left\|\Gamma_{\Sigma}^{t}(f)\right\| & \leq H(\tau)\|f\|,
\end{aligned}
$$

for $\tau=2 t J$ and for some analytic functions $G$ and $H$, which depend on $r$ but not on the length of $\Sigma$.

It is immediately clear from Theorem 21 and Corollary 22 that if $f$ is quasi-local then $\Gamma_{\Sigma}(f)$ is also quasi-local for every $t$, and its norm is bounded uniformly in the size of $\Sigma$.

\section{Expansionals}

In order to construct the operators required to prove Theorem 20, we will need the following object, which is known as a time-ordered exponential or expansional. For a detailed account of its properties see [60,61].

Definition 23 (Expansional [60, 61]). Let $O:[0,1] \rightarrow \mathcal{B}\left(\mathbb{C}^{D}\right)$ be a continuous path of operators such that

$$
\|O\|:=\sup _{t \in[0,1]}\|O(t)\|<\infty .
$$

The expansional of $O(t)$, also known as the time-ordered exponential of $O(t)$, is defined by

$$
\operatorname{Exp}\left[\int_{0}^{1} \mathrm{~d} t O(t)\right]:=\sum_{n=0}^{\infty} \int_{0}^{1} \mathrm{~d} t_{1} \int_{0}^{t_{1}} \mathrm{~d} t_{2} \cdots \int_{0}^{t_{n-1}} \mathrm{~d} t_{n} O\left(t_{1}\right) \cdots O\left(t_{n}\right) .
$$

Let us recall some useful properties of the expansional.

\section{Proposition 24.}

1. If $\left[O\left(t_{1}\right), O\left(t_{2}\right)\right]=0$ for all $t_{1}, t_{2} \in[0,1]$, then $\operatorname{Exp}\left[\int_{0}^{1} \mathrm{~d} t O(t)\right]$ is equal to the usual exponential of the integral $\exp \left(\int_{0}^{1} \mathrm{~d} t O(t)\right)$.

2. The norm of $\operatorname{Exp}\left[\int_{0}^{1} \mathrm{~d} t O(t)\right]$ is bounded by $\exp (\|O\|)$, and moreover it holds that

$$
\left\|\operatorname{Exp}\left[\int_{0}^{1} \mathrm{~d} t O(t)\right]-\mathbb{1}\right\| \leq \exp (\|O\|)-1
$$


3. 61. Proposition 15] Let $O_{1}, \ldots, O_{n}$ be operators. Then it holds that

$$
e^{O_{1}} \cdots e^{O_{n}}=\operatorname{Exp}\left[\int_{0}^{1} \mathrm{~d} t \sum_{m=1}^{n} \Gamma_{O_{1}}^{t} \circ \cdots \circ \Gamma_{O_{m-1}}^{t}\left(O_{m}\right)\right] .
$$

Proof of 2. Since we have that

$$
\operatorname{Exp}\left[\int_{0}^{1} \mathrm{~d} t O(t)\right]=\mathbb{1}+\sum_{n=1}^{\infty} \int_{0}^{1} \mathrm{~d} t_{1} \int_{0}^{t_{1}} \mathrm{~d} t_{2} \cdots \int_{0}^{t_{n-1}} \mathrm{~d} t_{n} O\left(t_{1}\right) \cdots O\left(t_{n}\right),
$$

then we can bound the norm of $\operatorname{Exp}\left[\int_{0}^{1} \mathrm{~d} t O(t)\right]-\mathbb{1}$ as follows:

$$
\begin{aligned}
\left\|\operatorname{Exp}\left[\int_{0}^{1} \mathrm{~d} t O(t)\right]-\mathbb{1}\right\| & \leq \sum_{n=1}^{\infty} \int_{0}^{1} \mathrm{~d} t_{1} \int_{0}^{t_{1}} \mathrm{~d} t_{2} \cdots \int_{0}^{t_{n-1}} \mathrm{~d} t_{n}\|O\|^{n} \\
& =\sum_{n=1}^{\infty} \frac{\|O\|^{n}}{n !}=\exp (\|O\|)-1 .
\end{aligned}
$$

\section{The finite range Hamiltonian case}

We are now ready to prove Theorem 20 In order to show that the thermal states $\rho_{\partial A B C}$ are approximately factorizable, we will explicitly construct operators $\sigma_{\partial A B C}:=\Delta_{z b} \Delta_{a z}$, $\sigma_{\partial B}:=\Omega_{z c} \Omega_{d z}, \sigma_{\partial A B}:=\Omega_{z c} \Delta_{a z}$, and $\sigma_{\partial B C}:=\Delta_{z b} \Omega_{d z}$ that satisfy Equations (14) and (15).

To avoid excessive use of superscripts, we will denote the Hamiltonian associated to the different regions as follows:

$$
\begin{aligned}
& \rho_{\partial A B C}=e^{2 Q_{a x y b}}, \text { with } Q_{a x y b}=\sum_{Z \subset a x y b} q_{Z} \\
& \rho_{\partial A B}=e^{2 R_{a x y c}}, \text { with } \quad R_{a x y c}=\sum_{Z \subset a x y c} r_{Z} \\
& \rho_{\partial B C}=e^{2 S_{d x y b}}, \text { with } S_{d x y b}=\sum_{Z \subset d x y b} s_{Z} \\
& \rho_{\partial B}=e^{2 T_{d x y c}}, \text { with } T_{d x y c}=\sum_{Z \subset d x y c} t_{Z} .
\end{aligned}
$$

Given $\alpha$ one or more of the regions $a, b, c, d, x, y$, we will write $Q_{\alpha}=\sum_{Z \subseteq \alpha} Q_{Z}$ to mean all of the local Hamiltonian terms of $Q_{a x y b}$ that are strictly inside $\alpha \subseteq a x y b$. The terms intersecting two regions (say $\alpha$ and $\beta$ ) will be denoted $Q_{\partial \alpha \beta}$. The same convention will be used for the other three Hamiltonians (Eqns. $\left.\begin{array}{|c|c|c|c|}43 & 44 & 46 & \text { ) }\end{array}\right)$.

We define the operators $\Delta$ and $\Omega$ as follows:

$$
\begin{aligned}
\Delta_{a x y} & :=e^{Q_{a x}} e^{-Q_{y}} e^{Q_{a x y}} \\
\Omega_{x y c} & :=e^{T_{x y c}} e^{-T_{x}} e^{T_{y c}} .
\end{aligned}
$$

We will use the properties of expansionals to show that, with these definitions of $\Delta$ and $\Omega$, the boundary states are approximately factorizable. We will first prove the following lemma, regarding $\sigma_{A B}$ : the rest of the bounds of Equations (14) and (15) can be proven in an analogous manner. 
Lemma 25. With the definitions given above, we have that

$$
\left\|\rho_{\partial A B}^{1 / 2} \sigma_{\partial A B}^{-1} \rho_{\partial A B}^{1 / 2}-\mathbb{1}\right\| \leq \eta(\epsilon(\ell))
$$

where $\eta(t)=e^{t}-1$ and

$$
\epsilon(\ell) \leq 2 F_{r}(2 J)\left[2 r\left(1+H(2 J)^{2}\right)+H(2 J) G(2 J)\right] \max \left\{J \chi_{\ell}(2 J), \sum_{l \geq \ell} \delta(l)\right\},
$$

and the functions $F_{r}, G, H$ and $\chi$ are given by Theorem 21 and Corollary 22

Proof. To start with, rewrite the expression

$$
\begin{aligned}
\rho_{\partial A B}^{1 / 2} \sigma_{\partial A B}^{-1} \rho_{\partial A B}^{1 / 2} & =e^{R_{a x y c}} \Delta_{a x y}^{-1} \Omega_{x y c}^{-1} e^{R_{a x y b}} \\
& =e^{R_{a x y c}} e^{-Q_{a x y}} e^{Q_{y}} e^{-Q_{a x}} e^{-T_{y c}} e^{T_{x}} e^{-T_{x y c}} e^{R_{a x y c}} \\
& =\left(e^{R_{a x y c}} e^{-Q_{a x y}} e^{Q_{y}} e^{-T_{y c}}\right)\left(e^{-Q_{a x}} e^{T_{x}} e^{-T_{x y c}} e^{R_{a x y c}}\right) \\
& :=O_{L} O_{R},
\end{aligned}
$$

where we have used that $Q_{a x}$ and $T_{y c}$ commute since they are non-overlapping. We will now show that there exists a decaying function $\epsilon(\ell)$, that we will specify later, such that

$$
\max \left(\left\|O_{L}-\mathbb{1}\right\|,\left\|O_{R}-\mathbb{1}\right\|\right) \leq \exp \left(\frac{\epsilon(\ell)}{2}\right)-1,
$$

which implies that

$$
\left\|O_{L} O_{R}-\mathbb{1}\right\| \leq\left\|O_{L}\right\|\left\|O_{R}-\mathbb{1}\right\|+\left\|O_{L}-\mathbb{1}\right\| \leq \exp (\epsilon(\ell))-1 .
$$

Now, in order to show that $O_{L}$ (or equivalently $O_{R}$ ) is close to the identity, we will apply equation (42) to the operator $O_{L}$ and obtain

$$
\begin{aligned}
& O_{L}=e^{R_{a x y c}} e^{-Q_{a x y}} e^{Q_{y}} e^{-T_{y c}}= \\
& \operatorname{Exp}\left[\int_{0}^{1} \mathrm{~d} t R_{a x y c}-\Gamma_{R_{a x y c}}^{t}\left(Q_{a x y}\right)+\Gamma_{R_{a x y c}}^{t} \Gamma_{Q_{a x y}}^{-t}\left(Q_{y}\right)-\Gamma_{R_{a x y c}}^{t} \Gamma_{Q_{a x y}}^{-t} \Gamma_{Q_{y}}^{t}\left(T_{y c}\right)\right] .
\end{aligned}
$$

We will further decompose the r.h.s. of the previous equation as follows: we substitute $R_{a x y c}=\Gamma_{R_{a x y c}}^{t}\left(R_{a x y c}\right)$ with $\Gamma_{R_{a x y c}}^{t}\left(R_{a x y}+R_{c}+R_{\partial y c}\right)$, and $\Gamma_{Q_{y}}^{t}\left(T_{y c}\right)$ with $\Gamma_{Q_{y}}^{t}\left(T_{y}+\right.$ $\left.T_{\partial y c}\right)+T_{c}$, since $\left[T_{c}, Q_{y}\right]=0$. The expression then reduces to

$$
O_{L}=\operatorname{Exp}\left[\int_{0}^{1} \mathrm{~d} t \sum_{i=1}^{5} X_{i}(t)\right]
$$

where

$$
\begin{array}{lll}
X_{1}(t) & =\Gamma_{R_{a x y c}}^{t}\left(R_{a x y}-Q_{a x y}\right), & X_{2}(t)=\Gamma_{R_{a x y c}}^{t}\left(R_{c}-T_{c}\right), \\
X_{3}(t)=\Gamma_{R_{a x y c}}^{t} \Gamma_{Q_{a x y}}^{-t} \Gamma_{Q_{y}}^{t}\left(Q_{y}-T_{y}\right), & X_{4}(t)=\Gamma_{R_{a x y c}}^{t} \Gamma_{Q_{a x y}}^{-t} \Gamma_{Q_{y}}^{t}\left(R_{\partial y c}-T_{\partial y c}\right), \\
X_{5}(t)=\Gamma_{R_{a x y c}}^{t}\left(R_{\partial y c}-\Gamma_{Q_{a x y}}^{-t} \Gamma_{Q_{y}}^{t}\left(R_{\partial y c}\right)\right) . &
\end{array}
$$


Let us denote $\epsilon(\ell):=2 \sum_{i}\left\|X_{i}\right\|$. Then by equation (41) we have that

$$
\left\|O_{L}-\mathbb{1}\right\| \leq \exp \left(\left\|\sum_{i} X_{i}\right\| \|\right)-1 \leq \exp \left(\frac{\epsilon(\ell)}{2}\right)-1 .
$$

Thus in order to bound $\left\|O_{L}-\mathbb{1}\right\|$, it remains to show that for each $i$ the norm $\left\|X_{i}\right\|$ is small. The first term can be bounded as follows. Note that

$$
R_{a x y}-Q_{a x y}=\sum_{Z \subset a x y}\left(r_{Z}-q_{Z}\right) .
$$

Then $\left(r_{Z}-q_{Z}\right)$ is zero if $Z$ has radius larger than $r$. Then, from Theorem 21, we get that $\Gamma_{R_{a x y c}}^{t}$ acting on a local operator is quasi-local in the sense of Def. 18 and that its norm is bounded by a constant function $F$, hence

$$
\left\|\Gamma_{R_{a x y c}}^{t}\left(R_{a x y}-Q_{a x y}\right)\right\| \leq F_{r}(2 t J) \sum_{Z \subset a x y}\left\|r_{Z}-q_{Z}\right\| \leq F_{r}(2 t J) \sum_{Z \subset a x y} \delta(d(Z, \partial C)),
$$

where term on the r.h.s. is controlled by $\delta$-homogeneity. Since any $Z \subset$ axy is at least at distance $\ell$ from $\partial C$, we obtain the bound:

$$
\left\|X_{1}\right\| \leq r F_{r}(2 J) \sum_{l>\ell} \delta(l) .
$$

A similar argument works for $\left\|X_{2}\right\|$, which can be bounded in the same way. In order to bound the norm of $X_{3}$ and $X_{4}$, we will have to add an extra step instead. We start from the same decomposition

$$
X_{3}(t)=\sum_{Z \subset y} \Gamma_{R_{a x y c}}^{t} \Gamma_{Q_{a x y}}^{-t} \Gamma_{Q_{y}}^{t}\left(q_{Z}-t_{Z}\right)
$$

where the sum only runs over $Z$ with diameter smaller than the interaction length $r$. Each of the three $\Gamma^{t}$ maps quasi-local operators to quasi-local operators, with a bound on the norm given by equation [35, so that we get

$$
\left\|\Gamma_{R_{a x y c}}^{t} \Gamma_{Q_{a x y}}^{-t} \Gamma_{Q_{y}}^{t}\left(q_{Z}-t_{Z}\right)\right\| \leq F_{r}(2 t J) H(2 t J)^{2} \delta(d(Z, \partial B)),
$$

so that again, we can bound

$$
\left\|X_{3}\right\| \leq r F_{r}(2 J) H(2 J)^{2} \sum_{l>\ell} \delta(l) .
$$

A similar analysis will work for $X_{4}$.

We now focus on $X_{5}$. We expect $\Gamma_{Q_{a x y}}^{-t} \Gamma_{Q_{y}}^{t}\left(R_{\partial y c}\right) \approx \Gamma_{Q_{y}}^{-t} \Gamma_{Q_{y}}^{t}\left(R_{\partial y c}\right)=R_{\partial y c}$ when $y$ is large enough. Once again, we use Theorem[21 and Corollary 22 to show that this indeed holds. As for the other terms, we invoke the fact that quasi-local operators get mapped to quasi-local operators under $\Gamma^{t}$. Furthermore, we note that since $R_{\partial y c}$ is strictly local we get that $R_{\partial y c}-\Gamma_{Q_{a x y}}^{-t} \Gamma_{Q_{y}}^{t}\left(R_{\partial y c}\right)$ is quasi-local with center at $\partial y c$. This implies that

$$
\left\|\Gamma_{R_{a x y c}}^{t}\left(R_{\partial y c}-\Gamma_{Q_{a x y}}^{-t} \Gamma_{Q_{y}}^{t}\left(R_{\partial y c}\right)\right)\right\| \leq H(2 t J)\left\|R_{\partial y c}-\Gamma_{Q_{a x y}}^{-t} \Gamma_{Q_{y}}^{t}\left(R_{\partial y c}\right)\right\|,
$$


by Theorem 21 We now set out to show that $\left\|R_{\partial y c}-\Gamma_{Q_{a x y}}^{-t} \Gamma_{Q_{y}}^{t}\left(R_{\partial y c}\right)\right\|$ is small:

$$
\begin{aligned}
\left\|R_{\partial y c}-\Gamma_{Q_{a x y}}^{-t} \Gamma_{Q_{y}}^{t}\left(R_{\partial y c}\right)\right\| & =\left\|\left(\Gamma_{Q_{y}}^{-t}-\Gamma_{Q_{a x y}}^{-t}\right) \Gamma_{Q_{y}}^{t}\left(R_{\partial y c}\right)\right\| \\
& \leq F_{r}(2 t J) G(2 t J) J \chi_{\ell}(2 t J) .
\end{aligned}
$$

Putting all of the bits together, we get that

$$
\epsilon(\ell) \leq F_{r}(2 J)\left[2 r\left(1+H(2 J)^{2}\right)+H(2 J) G(2 J)\right] \max \left\{J \chi_{\ell}(2 J), \sum_{l \geq \ell} \delta(l)\right\} .
$$

\section{E. Extension to quasi-local interactions}

In the proof of Lemma 25. Theorem 21 and Corollary 22 played a crucial role. It is clear from the proof that the same approach will generalize to a larger class of interactions as long as one is able to generalize equations (37) and (38) to such Hamiltonians.

Abstractly, we can see Corollary 22 as a statement about two classes of operators: on the one hand we have the local operators $\mathcal{U}_{0}$ and the quasi-local ones $\mathcal{U}_{1}$. Then the result states that, for every $H \in \mathcal{U}_{0}$ and $f \in \mathcal{U}_{1}$, the imaginary time evolution $\Gamma_{H}^{t}(f)$ still belongs to $\mathcal{U}_{1}$ for all time $t$, and moreover that it can be well approximated by evolutions generated by a "truncation" of $H$ around the center of the support of $f$. In the proof of Lemma 25 we then apply the imaginary time evolution $\Gamma_{H}^{t}$ to the interaction terms of the Hamiltonians, exploiting the fact that $\mathcal{U}_{0} \subset \mathcal{U}_{1}$.

Extensions of Corollary 22 would then enlarge the classes $\mathcal{U}_{0}$ and $\mathcal{U}_{1}$ for which these properties hold. By using similar techniques to the original proof, we believe that a first extension of Araki's theorem can be proven, to allow for quasi-local Hamiltonian interactions (so that $\mathcal{U}_{0}=\mathcal{U}_{1}$ are the quasi-local operators defined in Definition 18).

Conjecture 26 (Quasi-local Araki). Let $Q_{\Sigma}=\sum_{Z \subset \Sigma} q_{Z}$ be a quasi-local Hamiltonian on $\Sigma$ with strength $J$, and let $f$ be a quasi-local observable with center at the origin. Let $Q_{\ell}=\sum_{Z \subset[-\ell, \ell]} q_{Z}$. Then

$$
\begin{aligned}
\left\|\Gamma_{\Sigma}^{t}(f)-\Gamma_{\ell}^{t}(f)\right\| & \leq \mu_{\ell}(\tau) G(\tau)\|f\| \\
\left\|\Gamma_{\Sigma}^{t}(f)\right\| & \leq H(\tau)\|f\|,
\end{aligned}
$$

for $\tau=2 J t$ and for some analytic functions $G$ and $H$ which do not depend on the length of $\Sigma$.

We do not provide a proof of this generalization of Theorem 21 but we observe that if it holds, then the proof of Theorem 20 carries through verbatim of the case of quasi-local interactions, obtaining the following:

Theorem 27. If Conjecture 26 holds, then thermal states of quasilocal Hamiltonians with strength $J$ and $\delta$-homogeneous are $\epsilon(\ell)$-approximately factorizable on regions $\{\partial A B C, \partial A B, \partial B C, \partial B\}$, with

$$
\epsilon(\ell)=c(J)\left[\mu_{\ell}(2 J)+\sum_{l \geq \ell} \delta(l)\right]
$$

with $c(J)$ a positive constants independent of $\ell$. 


\section{F. Boundary Hamiltonians of PEPS}

We now comment on how to apply Theorem 20 and Theorem 27 in order to show that the boundary state of a PEPS is approximately factorizabile. If the PEPS is injective on region $A$, then the boundary state $\rho_{\partial A}$ will be full rank and therefore can be written as the Gibbs state of some Hermitian operator, which we will call the boundary Hamiltonian.

Definition 28. If a PEPS is injective on a region $A$, then the boundary Hamiltonian is given by

$$
Q_{\partial A}=\frac{1}{2} \log \left(\rho_{\partial A}\right),
$$

where $\rho_{\partial A}$ is the boundary state of the PEPS.

To cover the case of $G$-injective or MPO-injective PEPS, one faces the problem that, in such cases, boundary states are no longer full rank and hence cannot be Gibbs states of Hamiltonians. What is then the structure that is expected to hold in the boundary state of a gapped $G$-injective or MPO-injective PEPS? Numerical evidence from [29] and analytical evidence from [17] suggests that the boundary states are, in that case, of the form $J_{\partial A} e^{2 Q_{\partial A} \text {, }}$ where $J_{\partial A}$ is the MPO projector on the boundary of region $A$ and the boundary Hamiltonian $Q_{\partial A}$ is (quasi-)local, (quasi-)homogeneous and its constituent interactions all commute with $J_{\partial A}$.

We can then modify Definition 28 as follows.

Definition 29. If a PEPS is MPO-injective on a region $A$, and $J_{\partial A}$ is the projector on the kernel of $\rho_{\partial A}$, then the boundary Hamiltonian is given by

$$
Q_{\partial A}=\frac{1}{2} \log \left(\rho_{\partial A}\right) \in J_{\partial A} \mathcal{H}_{\partial A} J_{\partial A} ;
$$

where the logarithm is understood to be restricted to the support of $\rho_{\partial A}$.

Does the boundary Hamiltonian of a PEPS defined in this way satisfy the assumptions of Theorem 20? While we do not have a satisfying answer except for the case of isometric and G-isometric PEPS (for which the parent Hamiltonian is commuting), we comment on what numerical evidence can tell us. The range of the boundary Hamiltonian has been investigated in detail in Ref. [26] for the square lattice AKLT and the Ising PEPS models on a cylinder. There, the authors numerically compared the boundary Hamiltonians on the cylinder to the long range Heisenberg Hamiltonian:

$$
H=\sum_{\ell \geq 1} \eta_{\ell} \sum_{j \in \lambda} S_{j} S_{j+\ell}+R,
$$

where $R$ is some unknown rest term. They extracted the values of $\eta_{\ell}$ for a depth two and for an infinite depth cylinder, and found to very high accuracy that the terms $\eta_{\ell}$ decayed exponentially with $\ell$ for $\ell>2$. The norm of $R$ was also shown to be very small. They perform the same numerics for Ising PEPS in the non-critical regime, and also observe that the boundary Hamiltonian shows some decay in the interaction strength. In the regime where the Ising PEPS becomes non-ergodic, they find that the boundary state begins to resemble a mean field Heisenberg model; i.e. $\eta_{\ell}=O(1)$ for all $\ell$. While it is not reasonable to expect a finite range boundary Hamiltonian from these results, it seems quite challenging to distinguish a faster than exponential decay from an exponential decay, meaning that even assuming that the quasi-local version of Araki given in Conjecture 26 holds, one could not easily apply Theorem 27 
Unfortunately, the authors of Ref. [26] did not make any specific statements about the homogeneity of the boundary state, however there is some evidence in their numerics to support our assumptions. In [26, Fig. 8a] the magnitude of the $\eta_{\ell}$ term was plotted for different cylinder diameters. The values of $\eta_{\ell}$ are essentially independent of the cylinder diameter as long as $\ell<L / 2$, where $L$ is the cylinder diameter. This suggests, at least in the translationally invariant case, that the boundary Hamiltonian has a universal character, and only the (exponentially suppressed) very long range contributions are perturbed with changes at a long distance.

Beyond the numerical evidence above, locality of the boundary state has been shown to hold analytically for models of non-interacting free-fermions [62], and for certain conformal field theories [63]. However, caution must be taken, since systems with a chiral symmetry give rise to critical boundary states.

\section{CONCLUSION}

We have proved a fundamental theorem relating boundary states of two dimensional injective PEPS to the bulk gap of the parent Hamiltonian. Our work raises a lot of further questions for our understanding and analysis of bulk-boundary correspondences in many body systems as well as for relating static to dynamic properties in physical systems, which we discuss below.

a. Is the approximate factorization condition necessary? The most pressing question is perhaps to know if the assumptions on the boundary states made in Theorem 10, which are sufficient to prove a spectral gap in the bulk, are also necessary. There are good reasons to believe that this is the case. It was recently shown [64] that a uniform bulk gap of the parent Hamiltonian which is similar (although not identical) to Definition 5 implies that the PEPS satisfies local indistinguishability. Local indistinguishability, and its topological variant, LTQO [65], imply that local observables can be evaluated accurately by only contracting a finite ring of tensors around the observable. We believe (although we do not have a proof at present) that the property of LTQO should allow us to show that shielded regions of the boundary states satisfy the decay of mutual information bound that has recently been shown to by equivalent to the existence of local recovery maps [66]. This in turn implies that the boundary state is close to a local (although not necessarily bounded) Gibbs state [67]. Given this insight, as well as the numerical evidence from [26], we conjecture the following:

Conjecture 30. If for any rectangular region $A \subset \Lambda, H_{A}$ is gapped, then the boundary states of $A$ are close to a Gibbs state of a $1 D$ Hamiltonian with exponentially decaying interactions, and they are approximately factorizable.

More abstractly, one might ask whether there are further correspondences between bulk and boundary properties, including conventional symmetries. One interesting direction to look into is whether the effective temperature of the boundary Hamiltonian is related to the correlation length of bulk observables as predicted by Poilblanc [68].

b. A canonical form for PEPS? In every PEPS there is a gauge degree of freedom in its defining tensor, in the sense that if we multiply the left and right virtual levels by $Y$ and $Y^{-1}$ respectively, this action gets canceled in the tensor contraction that defines the PEPS. The same happens for the top and bottom virtual levels when multiplying by $Z$ and $Z^{-1}$ respectively. Moreover, by considering non-translation invariant tensor networks it is possible to change the choice of gauge matrices at each edge. While this operation does not change the physical state represented by the PEPS, it will transform the boundary state via a product of congruence (but not similarity) transformations, in the sense that the boundary state $\rho_{\partial A}$ of a region $A$ will be mapped to $\left(X_{1}^{\dagger} \otimes \cdots \otimes X_{|\partial A|}^{\dagger}\right) \rho_{\partial A}\left(X_{1} \otimes \cdots \otimes X_{|\partial A|}\right)$, 
where $\left\{X_{i}\right\}_{i}$ are invertible matrices. It has been proved in [69] that for injective PEPS this is the only freedom in the PEPS tensor. It is not clear however how to choose the best gauge matrices for a given PEPS. In 1D, the canonical form [54] defined in Lemma 12 gives a way to fix the gauge which implies the required approximate factorization of the boundary state. Based on this, one could then define the canonical gauge in 2D exactly as the one needed to have an approximate factorization of the boundary state (in case such factorization exists). Note that since the gauge transformation can potentially change both the eigenvalues and the eigenvectors of the boundary state, it is not clear that having the approximate factorization property for a given choice of gauge implies the same for other choices of gauges (given that square roots of the boundary states appear in (14) and (15)).

\section{Acknowledgments}

We thank Albert Werner and Wojciech De Roeck for fruitful discussions. M. J. K. was supported by the VILLUM FONDEN Young Investigator Program. A. L. acknowledges financial support from the European Research Council (ERC Grant Agreement no 337603), the Danish Council for Independent Research (Sapere Aude), VILLUM FONDEN via the QMATH Centre of Excellence (Grant No. 10059), the Walter Burke Institute for Theoretical Physics in the form of the Sherman Fairchild Fellowship as well as support from the Institute for Quantum Information and Matter (IQIM), an NSF Physics Frontiers Center (NFS Grant PHY-1733907). D. P. G. acknowledges support from MINECO (grant MTM2014-54240-P), Comunidad de Madrid (grant QUITEMAD+-CM, ref. S2013/ICE-2801), and Severo Ochoa project SEV-2015-556. This project has received funding from the European Research Council (ERC) under the European Union's Horizon 2020 research and innovation programme (grant agreement No 648913).

[1] Huzihiro Araki. Gibbs states of a one dimensional quantum lattice. Communications in Mathematical Physics, 14(2):120-157, 1969.

[2] Matthew B. Hastings. Solving gapped Hamiltonians locally. Physical Review B, 73(8):085115, 2006.

[3] Matthew B. Hastings. An area law for one-dimensional quantum systems. Journal of Statistical Mechanics: Theory and Experiment, 2007(08):P08024, 2007.

[4] Itai Arad, Alexei Kitaev, Zeph Landau, and Umesh Vazirani. An area law and sub-exponential algorithm for 1D systems, 2013. pre-print, arXiv:1301.1162 [quant-ph].

[5] Fernando G.S.L. Brandão and Michał Horodecki. Exponential decay of correlations implies area law. Communications in Mathematical Physics, 333(2):761-798, 2015.

[6] Andras Molnar, Norbert Schuch, Frank Verstraete, and J. Ignacio Cirac. Approximating Gibbs states of local Hamiltonians efficiently with projected entangled pair states. Physical Review B, 91(4):045138, 2015.

[7] Román Orús. A practical introduction to tensor networks: Matrix product states and projected entangled pair states. Annals of Physics, 349:117-158, 2014.

[8] Itai Arad, Zeph Landau, Umesh Vazirani, and Thomas Vidick. Rigorous RG Algorithms and Area Laws for Low Energy Eigenstates in 1D. Communications in Mathematical Physics, 356(1):65105, November 2017.

[9] Brenden Roberts, Thomas Vidick, and Olexei I. Motrunich. Implementation of rigorous renormalization group method for ground space and low-energy states of local hamiltonians. Physical Review B, 96(21), December 2017.

[10] Frank Pollmann, Ari M. Turner, Erez Berg, and Masaki Oshikawa. Entanglement spectrum of a topological phase in one dimension. Physical Review B, 81(6):064439, 2010. 
[11] Norbert Schuch, David Pérez-García, and Ignacio Cirac. Classifying quantum phases using matrix product states and projected entangled pair states. Physical Review B, 84(16):165139, 2011.

[12] Xie Chen, Zheng-Cheng Gu, and Xiao-Gang Wen. Classification of gapped symmetric phases in one-dimensional spin systems. Physical Review B, 83(3):035107, 2011.

[13] Lukasz Fidkowski and Alexei Kitaev. Topological phases of fermions in one dimension. Physical Review B, 83(7):075103, 2011.

[14] Norbert Schuch, Ignacio Cirac, and David Pérez-García. PEPS as ground states: degeneracy and topology. Annals of Physics, 325(10):2153-2192, 2010.

[15] Oliver Buerschaper. Twisted injectivity in projected entangled pair states and the classification of quantum phases. Annals of Physics, 351:447-476, 2014.

[16] Mehmet Burak Şahinoğlu, Dominic Williamson, Nick Bultinck, Michael Mariën, Jutho Haegeman, Norbert Schuch, and Frank Verstraete. Characterizing topological order with matrix product operators, 2014. pre-print, arXiv:1409.2150 [quant-ph].

[17] J. Ignacio Cirac, David Perez-Garcia, Norbert Schuch, and Frank Verstraete. Matrix product density operators: Renormalization fixed points and boundary theories. Annals of Physics, 378:100 $149,2017$.

[18] Nick Bultinck, Michael Mariën, Dominic J. Williamson, Mehmet B. Şahinoğlu, Jutho Haegeman, and Frank Verstraete. Anyons and matrix product operator algebras. Annals of Physics, 378:183233, 2017.

[19] Norbert Schuch, Didier Poilblanc, J. Ignacio Cirac, and David Pérez-García. Resonating valence bond states in the PEPS formalism. Physical Review B, 86(11):115108, 2012.

[20] Philip W. Anderson. Resonating valence bonds: A new kind of insulator? Materials Research Bulletin, 8(2):153-160, 1973.

[21] Brian Swingle. Entanglement renormalization and holography. Physical Review D, 86(6), September 2012.

[22] Fernando Pastawski, Beni Yoshida, Daniel Harlow, and John Preskill. Holographic quantum errorcorrecting codes: Toy models for the bulk/boundary correspondence. Journal of High Energy Physics, 2015(6):1-55, 2015.

[23] Patrick Hayden, Sepehr Nezami, Xiao-Liang Qi, Nathaniel Thomas, Michael Walter, and Zhao Yang. Holographic duality from random tensor networks. Journal of High Energy Physics, 2016(11):9, 2016.

[24] Isaac H. Kim and Michael J. Kastoryano. Entanglement renormalization, quantum error correction, and bulk causality. Journal of High Energy Physics, 2017(4):40, 2017.

[25] Hui Li and F. Duncan M. Haldane. Entanglement spectrum as a generalization of entanglement entropy: Identification of topological order in non-abelian fractional quantum hall effect states. Physical Review Letters, 101(1):010504, 2008.

[26] J. Ignacio Cirac, Didier Poilblanc, Norbert Schuch, and Frank Verstraete. Entanglement spectrum and boundary theories with projected entangled-pair states. Physical Review B, 83(24):245134, 2011.

[27] Frank Verstraete, Michael M. Wolf, David Perez-Garcia, and J. Ignacio Cirac. Criticality, the area law, and the computational power of projected entangled pair states. Physical Review Letters, 96(22):220601, 2006.

[28] Didier Poilblanc, Norbert Schuch, David Pérez-García, and J. Ignacio Cirac. Topological and entanglement properties of resonating valence bond wave functions. Physical Review B, 86(1):014404, 2012.

[29] Norbert Schuch, Didier Poilblanc, J. Ignacio Cirac, and David Perez-Garcia. Topological order in the projected entangled-pair states formalism: Transfer operator and boundary Hamiltonians. Physical Review Letters, 111(9):090501, 2013.

[30] Didier Poilblanc. Entanglement Hamiltonian of the quantum Néel state. Journal of Statistical Mechanics: Theory and Experiment, 2014(10):P10026, 2014.

[31] Manuel Rispler, Kasper Duivenvoorden, and Norbert Schuch. Long-range order and symmetry breaking in projected entangled-pair state models. Physical Review B, 92(15):155133, 2015.

[32] Olivier Gauthé and Didier Poilblanc. Entanglement properties of the two-dimensional SU(3) Affleck-Kennedy-Lieb-Tasaki state. Physical Review B, 96(12), September 2017.

[33] Bruno Nachtergaele. The spectral gap for some spin chains with discrete symmetry breaking. Communications in Mathematical Physics, 175(3):565-606, 1996. 
[34] Stefan Knabe. Energy gaps and elementary excitations for certain VBS-quantum antiferromagnets. Journal of statistical physics, 52(3-4):627-638, 1988.

[35] Ian Affleck, Tom Kennedy, Elliott H. Lieb, and Hal Tasaki. Valence bond ground states in isotropic quantum antiferromagnets. Communications in Mathematical Physics, 115:477-528, 1988.

[36] Toby S Cubitt, David Perez-Garcia, and Michael M. Wolf. Undecidability of the spectral gap. Nature, 528(7581):207-211, 2015.

[37] Shuo Yang, Thorsten B. Wahl, Hong-Hao Tu, Norbert Schuch, and J. Ignacio Cirac. Chiral Projected Entangled-Pair State with Topological Order. Physical Review Letters, 114(10), March 2015.

[38] J. Dubail and N. Read. Tensor network trial states for chiral topological phases in two dimensions and a no-go theorem in any dimension. Physical Review B, 92(20), November 2015.

[39] Didier Poilblanc, J. Ignacio Cirac, and Norbert Schuch. Chiral topological spin liquids with projected entangled pair states. Physical Review B, 91(22), June 2015.

[40] T. B. Wahl, H.-H. Tu, N. Schuch, and J. I. Cirac. Projected Entangled-Pair States Can Describe Chiral Topological States. Physical Review Letters, 111(23), December 2013.

[41] Michael A. Levin and Xiao-Gang Wen. String-net condensation: A physical mechanism for topological phases. Physical Review B, 71(4):045110, 2005.

[42] M.R. Norman. Colloquium: Herbertsmithite and the search for the quantum spin liquid. Reviews of Modern Physics, 88(4), December 2016.

[43] Lucile Savary and Leon Balents. Quantum spin liquids: a review. Reports on Progress in Physics, 80(1):016502, November 2016.

[44] Fabio Martinelli. Lectures on Glauber dynamics for discrete spin models. Lectures on probability theory and statistics, pages 93-191, 2004.

[45] Lorenzo Bertini, Nicoletta Cancrini, and Filippo Cesi. The spectral gap for a Glauber-type dynamics in a continuous gas. In Annales de l'IHP Probabilités et statistiques, volume 38, pages 91-108, 2002.

[46] Michael J Kastoryano and Angelo Lucia. Divide and conquer method for proving gaps of frustration free Hamiltonians. Journal of Statistical Mechanics: Theory and Experiment, (3):033105, 2018.

[47] Michael J. Kastoryano and Fernando G.S.L. Brandao. Quantum Gibbs Samplers: the commuting case. Communications in Mathematical Physics, pages 1-43, 2014.

[48] R.L. Dobrushin. Description of a random field by means of conditional probabilities, with applications. Teor. Veroyatnost. i Primenen, 13, 1968.

[49] O. E. Lanford and David Ruelle. Observables at infinity and states with short range correlations in statistical mechanics. Communications in Mathematical Physics, 13(3):194-215, 1969.

[50] Mark Fannes and R. F. Werner. Boundary conditions for quantum lattice systems. Helv. Phys. Acta, 68:635-657, 1995.

[51] Mark Fannes, Bruno Nachtergaele, and Reinhard F Werner. Finitely correlated states on quantum spin chains. Communications in Mathematical Physics, 144(3):443-490, 1992.

[52] Frank Verstraete, Valentin Murg, and J. Ignacio Cirac. Matrix product states, projected entangled pair states, and variational renormalization group methods for quantum spin systems. Advances in Physics, 57(2):143-224, 2008.

[53] Dorit Aharonov, Itai Arad, Umesh Vazirani, and Zeph Landau. The detectability lemma and its applications to quantum Hamiltonian complexity. New Journal of Physics, 13(11):113043, 2011.

[54] David Pérez García, F. Verstraete, M. M. Wolf, and J. I. Cirac. Matrix product state representations. Quantum Information \& Computation, 7(5):401-430, 2007.

[55] Michael M. Wolf. Quantum channels \& operations. guided tour. (accessed 2017-07-31), July 2012.

[56] Rajendra Bhatia. Matrix Analysis. Springer New York, 1997.

[57] Ola Bratteli and Derek W. Robinson. Operator Algebras and Quantum Statistical Mechanics 1. Springer Berlin Heidelberg, 1987.

[58] Elliott H. Lieb and Derek W. Robinson. The finite group velocity of quantum spin systems. Communications in Mathematical Physics, 28(3):251257, September 1972.

[59] Gabriel Bouch. Complex-time singularity and locality estimates for quantum lattice systems. Journal of Mathematical Physics, 56(12):123303, 2015.

[60] Izuru Fujiwara. Operator calculus of quantized operator. Progress of Theoretical Physics, 
7(5):433-448, 1952.

[61] Huzihiro Araki. Expansional in banach algebras. Annales scientifiques de l'École Normale Supérieure, 6(1):67-84, 1973.

[62] Lukasz Fidkowski. Entanglement spectrum of topological insulators and superconductors. Physical Review Letters, 104(13):130502, 2010.

[63] Jie Lou, Shu Tanaka, Hosho Katsura, and Naoki Kawashima. Entanglement spectra of the twodimensional Affleck-Kennedy-Lieb-Tasaki model: Correspondence between the valence-bondsolid state and conformal field theory. Physical Review B, 84(24):245128, 2011.

[64] M. Schwarz, O. Buerschaper, and J. Eisert. Approximating local observables on projected entangled pair states. Physical Review A, 95(6), June 2017.

[65] J. Ignacio Cirac, Spyridon Michalakis, David Pérez-García, and Norbert Schuch. Robustness in projected entangled pair states. Physical Review B, 88(11):115108, 2013.

[66] Omar Fawzi and Renato Renner. Quantum conditional mutual information and approximate Markov chains. Communications in Mathematical Physics, 340(2):575-611, 2015

[67] Kohtaro Kato and F.G.S.L. Brandao. Quantum Approximate Markov Chains are Thermal, 2016. pre-print, arxiv:1609.06636 [quant-ph].

[68] Didier Poilblanc. Entanglement spectra of quantum Heisenberg ladders. Physical Review Letters, 105(7):077202, 2010.

[69] D. Perez-Garcia, Mikel Sanz, C.E. Gonzalez-Guillen, Michael Marc Wolf, and J. Ignacio Cirac. Characterizing symmetries in a projected entangled pair state. New Journal of Physics, 12(2):025010, 2010. 